\title{
1 A microplate screen to estimate metal-binding affinities of 2 metalloproteins
}

Patrick Diep ${ }^{1}$, Radhakrishnan Mahadevan ${ }^{1,2}$ and Alexander F. Yakunin ${ }^{1,3}$

${ }^{1}$ BioZone Centre for Applied Bioscience and Bioengineering, Department of Chemical Engineering and Applied Chemistry, University of Toronto, Toronto, Ontario, Canada

${ }^{2}$ Institute of Biomaterials and Biomedical Engineering, University of Toronto, Toronto, Ontario, Canada

${ }^{3}$ Centre for Environmental Biotechnology, School of Natural Sciences, Bangor University, Bangor, United Kingdom

\section{Highlights}

- an improved version of a high-throughput screen (microlTFQ-LTA) is described for multiplexed elucidation of metalloprotein binding profiles

- validation was accomplished with the previously characterized CjNikZ; testing was accomplished with an uncharacterized homologue herein named CCSBPII

- CCSBPII is shown to bind to multiple transition metal ions with a large range of affinities, and potentially overcome mismetallation using a simple histidine metallophore

\begin{abstract}
Solute-binding proteins (SBPs) from ATP-binding cassette (ABC) transporters play crucial roles across all forms of life in transporting compounds against chemical gradients. Some SBPs have evolved to scavenge metal substrates from the environment with nanomolar and micromolar affinities $\left(K_{\mathrm{D}}\right)$. There exist well established techniques like isothermal titration calorimetry for thoroughly studying these metalloprotein interactions with metal ions, but they are low-throughput. For protein libraries comprised of many metalloprotein homologues and mutants, and for collections of buffer conditions and potential ligands, the throughput of these techniques is paramount. In this study, we describe an improved method termed the microlTFQ-LTA and validated it using CjNikZ, a well-characterized nickel-specific SBP (Ni-BP) from Campylobacter jejuni. We then demonstrated how the microlTFQ-LTA can be designed to screen through a small collection of buffers and ligands to elucidate the binding profile of a putative Ni-BP from Clostridium carboxidivorans that we call CCSBPII. Through this study, we showed CCSBPII can bind to various metal ions with $K_{\mathrm{D}}$ ranged over 3 orders of magnitude. In the presence of L-histidine, CcSBPII could bind to $\mathrm{Ni}^{2+}$ over 2000-fold more tightly, which was 11.6-fold tighter than CjNikZ given the same ligand.
\end{abstract}

\section{Keywords}

metalloprotein; solute-binding protein; $A B C$ transporter; metal-binding assay; tryptophan quenching

\section{Short Title}

A fluorescence-based microplate screen for metal-protein interactions 


\section{Introduction}

Metalloproteins are proteins whose function and structure involve interactions with metal ions. Several techniques in metal-binding studies can be used to study these interactions in vitro. The most widely accepted technique is isothermal titration calorimetry (ITC) as it can determine multiple thermodynamic parameters in a single binding experiment. However, obtaining meaningful ITC data is not a trivial undertaking. Requisites include an easily expressed protein in large quantities that is stable under prolonged stirring conditions (viz. it does not precipitate), the protein generates a heat signature upon binding that is appreciably larger than the heat of dilution, there is sufficient time to run multiple ITC trials to optimize the ratio of ligand and protein, and more. This last requirement can be especially cumbersome due to the time needed to equilibrate the system, but a value " $c$ " can be calculated to better adjust this ratio if an accurate estimation of the protein's binding constant $K_{\mathrm{D}}$ can be made using other techniques. ${ }^{1,2}$ Given that meaningful ITC data is obtained, one must consider whether ITC is the most appropriate technique to determine the binding constants for large protein families, mutant libraries, or both; and for multiple ligands and solvent conditions of interest. It is useful in such cases to consider a high-throughput screening technique, such as one that employs intrinsic tryptophan fluorescence quenching (ITFQ) in a ligand titration assay (LTA), herein termed ITFQ-LTA, to complement the lower throughput but more comprehensive ITC experiments.

Fluorescence quenching of a protein's intrinsic fluorophores (i.e. tryptophan) upon sequential exposure to a ligand through step-wise titration can generate binding curves for estimating $K_{\mathrm{D}}$, which therefore complements ITC by helping to calculate c. To increase the throughput for more extensive library screening, microplate readers equipped with fluorescence-reading capabilities can be used instead of spectrofluorometers, which has been tested for interactions between albumins and proteases with organic compounds. ${ }^{3-5}$ Microplate-based ITFQ-LTA (microlTFQ-LTA) has also been used to study interactions between metalloproteins and $\mathrm{UO}_{2}{ }^{2+}$, but these researchers' decision to use Equation (1), the Stern-Volmer equation (variables described further below), to fit the fluorescence quenching data is questionable. ${ }^{6,7}$

$$
\text { (1) } \frac{F_{0}-F}{F}=K_{s v}[L]=k_{q} \tau[L]_{a}
$$

The Stern-Volmer equation models collisional quenching, a phenomenon where the excited-state fluorophore is returned to ground state through contact with an external quencher in a non-radiative manner. This typically occurs via spin-orbit coupling with paramagnetic entities. ${ }^{8}$ Proteinaceous tryptophans are often partially or fully buried in the hydrophobic core where these external quenchers have limited access, and cannot easily collide. Consequently, static quenching is an unlikely mechanism for protein fluorescence quenching too because it requires the external quencher to form a complex with the ground-state proteinaceous tryptophan. ${ }^{9}$ A stronger explanation is intramolecular self-quenching. Tryptophan's large dipole moment causes it to be highly sensitive to changes in the polarity of its environment. Ligand-binding frequently induces conformational changes across the protein structure that changes the direction and magnitude of local electric fields around the tryptophans' indole rings. This can lead to saturable quenching (and sometimes enhancement) of its fluorescence through increased nonradiative energy transfer to nearby moieties of the surrounding protein matrix, typically through electron transfer into the peptide backbone and nearby side chains. ${ }^{9-14}$

Regardless of the exact mechanism for protein fluorescence quenching, the association phenomenon (i.e. binding event) can be followed by the magnitude of the change in fluorescence in response to the ligand titrations, modelled by Equation (2) for 1:1 metal-protein complexes. This model uses basic mass-action kinetics that assumes the protein and ligand concentration that can freely form 
complexes at any point in time during the ITFQ-LTA is a sum of what is uncomplexed (i.e. free) and what is already complexed, described in Equations (3) and (4). ${ }^{9,15,16}$

(2) $\frac{F_{0}-F}{F_{0}-F_{c}}=\frac{[P]_{t}+[L]_{a}+K_{\mathrm{D}}-\sqrt{\left([P]_{t}+[L]_{a}+K_{\mathrm{D}}\right)^{2}-4[P]_{t}[L]_{a}}}{2[P]_{t}}$

(3) $[L]_{a}=[L]_{\text {free }}+[P L]$

(4) $[P]_{t}=[P]_{\text {free }}+[P L]$

For all Equations in this study: $F_{0}$ and $F_{\mathrm{c}}$ are the measured fluorescence when the protein is fully unsaturated and fully saturated, respectively. $F$ is the measured fluorescence at a ligand titration step. $K_{\mathrm{sv}}$ is the Stern-Volmer quenching constant and $k_{\mathrm{q}}$ is the bimolecular quenching rate constant. $\tau$ is the fluorescence lifetime of the unquenched fluorophore. $[L]_{a}$ and $[P]_{t}$ are the concentrations of total ligand and protein, respectively. $[L]_{\text {free }}$ and $[P]_{\text {free }}$ are the concentrations of ligand and protein that are uncomplexed with each other, respectively. $[P L]$ is the concentration of the protein complexed with the ligand, and $K_{\mathrm{D}}$ is the apparent dissociation constant under a specific buffering condition.

Depending on how strongly the protein's tryptophan fluorophores respond to a binding event, which is based on the number of tryptophans in the protein and the extent of the ligand-induced conformational change, a large signal with low noise can be obtained for low micromolar protein concentrations. If the $K_{\mathrm{D}}$ for a ligand is below the protein concentration by at least a factor of 10 , it is generally more appropriate to use Equation (2). If the $K_{D}$ for a ligand is larger than the protein concentration, which means the range of ligand concentrations used in the microlTFQ-LTA is in molar excess of protein concentration, $[P L]$ can be neglected in Equation (3), which simplifies the mass-action kinetics underpinning Equation (2) to yield Equation (5). ${ }^{9}$

(5) $\frac{F_{0}-F}{F_{0}-F_{c}}=\frac{[L]_{a}}{K_{\mathrm{D}}+[L]_{a}}$

To simplify the variables for data analysis in this study, Equation (2) will be re-written as Equation (6), and Equation (5) will be re-written as Equation (7). $F_{\text {obs }}$ is $F_{0}-F$ and $F_{\max }$ is $F_{0}-F_{c}$. When the Hill Coefficient " $n$ " is used, $[L]_{a}$ in both Equations $(6)$ and $(\mathbf{7})$ is substituted with $[L]_{a}{ }^{n}$.

(6) $F_{\text {obs }}=\frac{F_{\max }\left([P]_{t}+[L]_{a}+K_{D}-\sqrt{\left([P]_{t}+[L]_{a}+K_{D}\right)^{2}-4[P]_{t}[L]_{a}}\right)}{2[P]_{t}}$

(7) $F_{\mathrm{obs}}=\frac{F_{\max }[L]_{a}}{K_{D}+[L]_{a}}$

One group of metalloproteins are the solute-binding protein (SBP) components of metal ATPbinding cassette $(A B C)$ transporters. These transporters are ubiquitous in nature as they provide cells the means to transport metal substrates against chemical gradients using the energy from nucleotide triphosphate hydrolysis. The specificity of $A B C$ transporters is derived from its cognate SBP that contains the binding site for target metal ions. ${ }^{17}$ NikA from the Escherichia coli $A B C$ transporter NikABCDE is the most thoroughly studied nickel-specific SBP (Ni-BP) with extensive biochemical and structural characterization. ${ }^{18-26}$ Other Ni-BPs have also been studied because of their implications in health, and include the pathogens Campylobacter jejuni (CjNikZ) ${ }^{27,28}$, Brucella suis $(B s N i k A)^{28,29}$, Yersinia pestis $(\text { YpYntA })^{28}$, Staphylococcus aureus (SaNikA, SaCntA $)^{30}$, Helicobacter hepaticus (HhNikA) $)^{31}$, Helicobacter pylori (CeuE) ${ }^{32}$, Vibrio parahaemolyticus $(\mathrm{VpNikA})^{33}$, and Clostridium difficile (CdOppA) $)^{34}$. However, it is not 
only pathogens that require metals to survive. Clostridium carboxidivorans is not known to be pathogenic, but possesses a cluster of coding sequences herein termed the acetogenesis/nickel acquisition (ana) operon that encodes a putative metal $A B C$ transporter and acetogenesis-related enzymes that use metal co-factors (Supplementary Figure 1). This ABC transporter possesses two SBPs, herein termed CcSBPI and CCSBPII, which are both homologous to CjNikZ (41\% and 37\%, respectively).

We are interested in studying and engineering Ni-BPs and their cognate $A B C$ transporters for mining applications. In this study, we took a different approach from the aforementioned $\mathrm{UO}_{2}{ }^{2+}$ metalloprotein binding study and instead used Equations (6) and (7) to fit our microlTFQ-LTA data. We first validated our method by closely replicating the $K_{\mathrm{D}}$ values for $\mathrm{Ni}^{2+} \rightarrow C j N i k Z$ that have previously been determined using spectrophotometer-based ITFQ-LTA (specITFQ-LTA) and ITC. We then studied $\mathrm{Ni}^{2+} \rightarrow$ CCSBPII with different buffers and varied the ligands afterwards. CCSBPII is an uncharacterized metalloprotein, but shares structural similarity to CjNikZ (Supplementary Figure 2), so we narrowed our scope to elucidating its binding profile using the microlTFQ-LTA screen so that it can be further studied with more comprehensive techniques like ITC and x-ray crystallography. Therefore, the focus of this study was to test and describe a microlTFQ-LTA screening method for metalloproteins that builds on previous iterations of the method. ${ }^{3-7}$ We report CCSBPII could bind to numerous transition metal ions with $K_{\mathrm{D}}$ values that ranged over 3 orders of magnitude, and could possibly deal with $\mathrm{Cu}^{2+}$ mismetallation by using Lhistidine as a simple metallophore to selectively bind to the theoretical target $\mathrm{Ni}^{2+}$.

\section{Materials and Methods}

DNA synthesis, cloning, and strains

The open-reading frames encoding CCSBPII from Clostridium carboxidivorans P7T (Ccar_RS11695, NCBI ID: NZ_CP011803.1) and CjNikZ ${ }^{28}$ were synthesized (Twist Bioscience, San Francisco, USA) without start codons and without their native signal peptides identified using SignalP 5.0 with the Gram-positive setting. ${ }^{35}$ Their open-reading frames were cloned into the expression vector p15TV-L (AddGene ID: 26093) under the T7 promoter and in-frame with the $\mathrm{N}$-terminal 6xHisTag (Twist Bioscience). Upon receiving the completed plasmids, they were transformed into the DH5 $\alpha$ and LOBSTR (Kerafast, \#EC1001) Escherichia coli strains, then plated onto LB-Agar (carbenicillin, $100 \mu \mathrm{g} / \mathrm{mL}$ ). The plasmids were miniprepped (GeneAid, \#PD300) into MilliQ water and sequence verified at the ACGT Sequencing Facility (Toronto, Canada). Glycerol stocks were prepared for storage at $-80^{\circ} \mathrm{C}$.

\section{Protein expression and purification}

Starter cultures for CjNikZ and CCSBPII were grown from glycerol stock in LB with carbenicillin (100 $\mu \mathrm{g} / \mathrm{mL}$ ) for $16 \mathrm{hrs}$ overnight at $37{ }^{\circ} \mathrm{C}$ with shaking. Expression cultures were started by pre-warming TB media with $5 \%$ glycerol and carbenicillin $(100 \mu \mathrm{g} / \mathrm{mL})$ to $37^{\circ} \mathrm{C}$ before $1 \% \mathrm{v} / \mathrm{v}$ inoculation with the starter culture, then grown for $3 \mathrm{hrs}$ at $37^{\circ} \mathrm{C}$ until addition of IPTG (BioShop, \#IPT002) to $0.4 \mathrm{mM}$ for induction. The expression cultures were then transferred to $16^{\circ} \mathrm{C}$ and grown for $16 \mathrm{hrs}$ overnight with shaking, then pelleted with centrifugation and transferred to conical vials for one freeze-thaw cycle at $-20^{\circ} \mathrm{C}$.

Frozen cell pellets were thawed and resuspended in binding buffer $(10 \mathrm{mM} \mathrm{HEPES}, 500 \mathrm{mM} \mathrm{NaCl}$, $5 \mathrm{mM}$ imidazole, $\mathrm{pH} 7.2$ ) to a final volume of $100 \mathrm{~mL}$, followed by addition of $0.25 \mathrm{~g}$ lysozyme (BioShop, \#LYS702). Cell pellet mixtures were sonicated for $25 \mathrm{~min}$ (Q700 Sonicator, Qsonica) and clarified by centrifugation. The soluble layer (supernatant) was applied to a cobalt-charged (Co-)NTA resin (ThermoFisher, \#88221) pre-equilibrated with binding buffer in a gravity-column set-up. Bound proteins were cleansed with wash buffer (10 mM HEPES, $500 \mathrm{mM} \mathrm{NaCl}, 25 \mathrm{mM}$ imidazole, pH 7.2) and the bound protein was collected with elution buffer (10 mM HEPES, $500 \mathrm{mM} \mathrm{NaCl}, 250 \mathrm{mM}$ imidazole, pH 7.2). 
Protein concentrations were determined by Bradford assay, and protein purity was determined by SDSPAGE analysis and densitometry on Image Lab 6.0 (Bio-Rad).

Eluted protein was combined with $0.4 \mathrm{mg}$ of in-house purified TEV protease and TCEP to $1 \mathrm{mM}$ (BioShop, \#TCE101), then transferred to a $10 \mathrm{kDa}$ MWCO dialysis bag (ThermoFisher, \#68100) for dialysis in $4 \mathrm{~L}$ dialysis buffer (10 mM HEPES, $1 \mathrm{mM} \mathrm{TCEP}, 1 \mathrm{~g} / \mathrm{L}$ Chelex 100, $\mathrm{pH} \mathrm{7.2)} \mathrm{at} 4{ }^{\circ} \mathrm{C}$ with gentle stirring for $72 \mathrm{hrs}$. Dialyzed samples were then applied to a cobalt-charged NTA resin twice and concentrated to $2 \mathrm{~mL}$ by centrifugation using $30 \mathrm{kDa}$ MWCO concentrators (Millipore Sigma, \#GE28-9323-61). This concentrate was applied to a MonoQ 5/50 anion exchange resin (GE Healthcare, \#17516601) using 10 mM HEPES, $\mathrm{pH} 7.2$ for the mobile phase, and $10 \mathrm{mM}$ HEPES, $1 \mathrm{M} \mathrm{NaCl}$, pH 7.2 for elution. Eluted proteins were collected in 96 deep-well blocks and stored at $4{ }^{\circ} \mathrm{C}$ during SDS-PAGE analysis. Selected fractions with sufficient purity were transferred to a $10 \mathrm{kDa}$ MWCO dialysis bag for dialysis in $4 \mathrm{~L}$ dialysis buffer (10 mM HEPES, pH 7.2) at $4{ }^{\circ} \mathrm{C}$ with gentle stirring for $24 \mathrm{hrs}$ and transferred to fresh dialysis buffer for another $24 \mathrm{hrs}$. Finally, they were flash-frozen drop-wise in liquid nitrogen before storage at $-80^{\circ} \mathrm{C}$. Prior to usage in preliminary microlTFQ-LTAs, the proteins were checked using circular dichroism, MALDI-ToF, and ICP-MS to ensure proper folding, molecular weight (i.e. successful 6x HisTag removal), and minimal metal contamination, respectively (Supplementary Information, Appendix A; Supplementary Figures 3 \& 4).

\section{Preparation of activity buffers and ligand solution}

Except for CjNikZ which used either 20mM MOPS pH 8 or 40 mM HEPES pH 7.5 as the activity buffer to mimic conditions used in prior studies ${ }^{27,28}$, CCSBPII used $10 \mathrm{mM}$ HEPES pH 7.2 as the default activity buffer with adjustments made as prescribed by the type of microITFQ-ITA (i.e. HEPES concentration range, $\mathrm{pH}$ range, $\mathrm{NaCl}$ range). Activity buffers were adjusted to the desired $\mathrm{pH}$ using $\mathrm{NaOH}$ and $\mathrm{HCl}$, then filter sterilized by syringe using a $0.2 \mu \mathrm{m}$ PTFE membrane disc (PALL ID: 4187). Ligand solutions were created using the appropriate activity buffer as prescribed by the type of microlTFQ-LTA to match the activity buffer in the well that the protein was diluted into (e.g. $1 \mathrm{mM} \mathrm{NiCl}_{2}$ was prepared in $10 \mathrm{mM}$ HEPES pH 7.2 from a $1 \mathrm{M}$ stock solution of $\mathrm{NiCl}_{2}$ dissolved in de-ionized water, in order to be added to CCSBPII diluted into $10 \mathrm{mM} \mathrm{HEPES} \mathrm{pH} \mathrm{7.2).} \mathrm{Metal} \mathrm{salts} \mathrm{with}>98 \%$ purity were used to prepare all ligand solutions requiring $\mathrm{NiCl}_{2}, \mathrm{NiSO}_{4}, \mathrm{CoCl}_{2}, \mathrm{CuCl}_{2}, \mathrm{ZnCl}_{2}, \mathrm{MnCl}_{2}$, and $\mathrm{CdCl}_{2}$. Ni-His $2, \mathrm{Co}-\mathrm{His}_{2}, \mathrm{Cu}-\mathrm{His}{ }_{2}$ were each prepared with a 1:2 stoichiometric ratio using the metal chloride salt and L-histidine. $\mathrm{Ni}_{-} \mathrm{Imi}_{2}, \mathrm{Ni}-\mathrm{Gly}$, and $\mathrm{Ni}$-Imi ${ }_{2} \mathrm{Gly}_{2}$ were prepared with a 1:2, 1:2, and 1:2:2 stoichiometric ratio using $\mathrm{NiCl}_{2}$, imidazole (imi), and glycine (gly). $\mathrm{Ni}_{2}$-Citrate ${ }_{2}, \mathrm{Ni}$-EDTA, and Ni-DETA 2 were prepared with a 2:2, 1:1, and 1:2 stoichiometric ratio. All ligand solutions were prepared fresh and immediately used in microlTFQ-LTAs; activity buffers were stored at $4^{\circ} \mathrm{C}$.

\section{Microplate-based intrinsic tryptophan fluorescence quenching - ligand titration assay (microlTFQ-LTA)}

microlTFQ-LTAs were performed in black, opaque 96-well microplates (Greiner Bio-One, \#655076) using the SpectraMax M2 spectrophotometer (Molecular Devices) with the following settings: top-read, $25^{\circ} \mathrm{C}, \lambda_{\mathrm{ex}} 280 \mathrm{~nm}, \lambda_{\mathrm{em}} 380 \mathrm{~nm}$, PMT high, sensitivity 30, carriage speed high, $5 \mathrm{~s}$ shaking before endpoint reads, and $3 \mathrm{~s}$ shaking in between reads during kinetic reads. CjNikZ and CCSBPII were combined with the appropriate activity buffer as prescribed by the specific microlTFQ-LTA to $0.4 \mu \mathrm{M}$ for a total volume of 200 $\mu \mathrm{L}$ and allowed to equilibrate with intermittent shaking for up to $15 \mathrm{~min}$, or until the signal stabilized. After equilibration, aliquots of the ligands are added in stepwise fashion using a multichannel pipette up to a maximum of $120 \mu \mathrm{L}$ when saturation was not reached, otherwise less. Mixing by pipette was avoided to prevent the introduction of air bubbles or the retaining of droplets inside the tips that would distort the signal. Therefore, each titration was followed by a $30 \mathrm{~s}$ equilibration with intermittent shaking before endpoint reads were made to ensure proper mixing. All trials were done in triplicates. To account for 
possible dilution effects in every trial, we included a triplicate negative control where the protein was titrated with only activity buffer (no metal ligand) using the same volumes added at each titration. A minor IFE was exhibited by histidine, which was accounted for by subtracting its individual fluorescence signal at $\lambda_{\text {em }} 380 \mathrm{~nm}$ (obtained by titrating histidine in the absence of metals or protein) from each titration step. Otherwise, all ligand solutions and components of activity buffers did not exhibit the IFE for the concentrations used.

225

226

227

\section{Data analysis and visualization}

All raw data is prepared in Microsoft 365 Excel for further analysis. Triplicate data for tests and negative controls (as described above) were averaged for each titration step (error propagation applied). The averaged negative control data was then subtracted from the averaged ligand test data at each titration step to remove the background signal and obtain $F$. The value of $F$ before any titration is made, where the protein is unsaturated, is $F_{0}$. To obtain a $F_{\text {obs }}$ value for each titration step, $F$ from all titration steps are subtracted from $F_{0}$ such that the new $F_{0}$ is zero and an up-right curve can be generated. These sets of $x / y$ data pairs (e.g. $[L]_{a} / F_{o b s}$, respectively) are inputted into GraphPad Prism 5.0 for determination of best-fit parameter values and confidence intervals through non-linear least-squares (NLLS) regression using Equation (6). This is performed with and without the hill coefficient $n$ as needed, where $[L]_{a}$ is substituted with $[L]_{a}{ }^{n}$. The Kemmer and Keller (KK) method, described elswhere ${ }^{36}$, is used in Excel to compute an NLLS regression using Equation (6) or (7) for determining best-fit parameter values and 95\% confidence intervals, including $\mathrm{n}$. In all NLLS regressions, the minimization parameter is $\chi^{2}$, in which the residuals are weighted by observed variability of $F_{\text {obs }}\left(1 / \sigma^{2}\right)$. All figures are produced using GraphPad Prism 5.0 (or for brevity, "Prism").

\section{Results and Discussion}

microITFQ-LTA closely replicates published binding constants for CjNikZ and supports crystal structure data

The Ni-BP CjNikZ belongs to NikZYXWV, a nickel ABC transporter, and has published $K_{\mathrm{D}}$ values of $0.61 \pm 0.07 \mu \mathrm{M}$ and $1.6 \pm 0.2 \mu \mathrm{M}$ (89\% difference), determined by a speclTFQ-LTA ${ }^{27}$ and by ITC $^{28}$, respectively. In these studies, non-linear least squares (NLLS) regressions were performed to obtain these best-fit parameter values with the commercial software packages SigmaPlot and MicroCal Origin. To fit the data, one-binding site models $(n=1)$ were used. In this study, we determined the $K_{\mathrm{D}}$ value of $C j$ NikZ in the same buffers used in these studies: $20 \mathrm{mM}$ MOPS pH 8, and $40 \mathrm{mM} \mathrm{HEPES} \mathrm{pH} \mathrm{7.5.} \mathrm{We} \mathrm{also} \mathrm{used} \mathrm{the}$ same nickel salt to prepare the ligand solutions: $\mathrm{NiCl}_{2}$ and $\mathrm{NiSO}_{4}$, respectively.

Assuming CjNikZ has one binding site $(n=1)$, and given that the concentration of nickel to be titrated is in molar excess of the protein concentration used $(0.4 \mu \mathrm{M})$, Equation (7) was chosen to model the data using Prism (Figure 1). In $20 \mathrm{mM} \mathrm{MOPS} \mathrm{pH} \mathrm{8,} \mathrm{we} \mathrm{find} \mathrm{that} \mathrm{Ni}^{2+} \rightarrow C j N i k Z$ yields a $K_{\mathrm{D}}$ value of 2.23 $\mu \mathrm{M}$ with a confidence interval $(\mathrm{Cl})$ of 1.53 to $2.92 \mu \mathrm{M}$. In $40 \mathrm{mM}$ HEPES pH 7.5, we find that $\mathrm{Ni}^{2+} \rightarrow C j \mathrm{NikZ}^{2}$ yields a $K_{\mathrm{D}}$ value of $3.34 \mu \mathrm{M}$ with a $\mathrm{Cl}$ of 2.41 to $4.26 \mu \mathrm{M}$. This difference in $K_{\mathrm{D}}$ values matches the difference in the published $K_{\mathrm{D}}$ in that the MOPS condition had a slightly lower $K_{\mathrm{D}}$ than the HEPES condition. Since crystallographic data shows that $C j N i k Z$ has only one binding site for $\mathrm{Ni}^{2+}$, we tested our first assumption here and included $\mathrm{n}$ in Equation (7) for a separate analysis. We found $\mathrm{n}$ to be 0.90 with a $\mathrm{Cl}$ of 0.60 to 1.21 , and similar $K_{\mathrm{D}}$ and $F_{\max }$ values, suggesting that the assumption is correct. Given that $C j N i k Z^{\prime} \mathrm{s}$ $K_{\mathrm{D}}$ value of $1.6 \mu \mathrm{M}$ determined by ITC is the most accurate value, the observed $K_{\mathrm{D}}$ values of $2.23 \mu \mathrm{M}$ and $3.34 \mu \mathrm{M}$ are only $33 \%$ and $70 \%$ different, respectively, which suggests the microlTFQ-LTA is as accurate as the specITFQ-LTA that yielded an $89 \%$ difference. 
While Prism can model data with Equation (7), it cannot be used with Equation (6) for scenarios where the $K_{\mathrm{D}}$ for a ligand is below the protein concentration in the assay because this requires the inclusion of a second independent variable $[P]_{t}$ that changes at each titration due to dilution. Therefore, we utilized a method described by Kemmer \& Keller (2010) that combines Excel's Solver function for NLLS regression with a simple plug-and-chug routine using Fisher's $F$ distribution to determine the Cls for the best-fit parameter values, herein termed the KK method for brevity. Using Equation (7) with the data for $\mathrm{Ni}^{2+} \rightarrow C j$ NikZ in $40 \mathrm{mM}$ HEPES pH 7.5, we found Prism and Solver's NLLS regression algorithms generated nearly identical best-fit parameters values (Table 1). Comparing the Cls computed by Prism and the KK method, we found they were also nearly identical, suggesting the KK method was a reliable protocol for modelling data with Equation (7) on Excel. Applying the KK method with Equation (6) instead for this data, we find there is little difference made to the best-fit parameter values, indicating that $[P]_{\mathrm{t}}$ was indeed negligible for characterizing this particular binding interaction between $\mathrm{Ni}^{2+}$ and $\mathrm{CjNikZ}$, thus confirming the assumption made earlier to choose Equation (7).

Interestingly, Cls could not be computed by the KK method for Equations (6) and (7) when $\mathrm{n}$ was included. Overparameterization can be diagnosed through the KK method using its $\chi^{2}$ plots. Here, a value $\chi^{2}$ bf is calculated using Fisher's $\mathrm{F}$ distribution and the bounds for the $95 \% \mathrm{Cl}$ of a best-fit parameter value are defined by where the curves have a $y$-value equal to $\chi^{2}$ bf, based on a polynomial function. Therefore, a requisite for the KK method to be used is for the slope of the curve on both sides to be sufficiently steep to define a reasonable $\mathrm{Cl}$. We generated $\chi^{2}$ plots against $K_{\mathrm{D}}$ and $F_{\max }$ (Supplementary Figure 5) for both models and found the inclusion of $n$ reduced $\chi^{2}$ by $4-6 \%$ (viz. marginally improved the goodness of fit), but

๑- 20 mM MOPS pH $8 \rightarrow 40$ mM HEPES pH 7.5
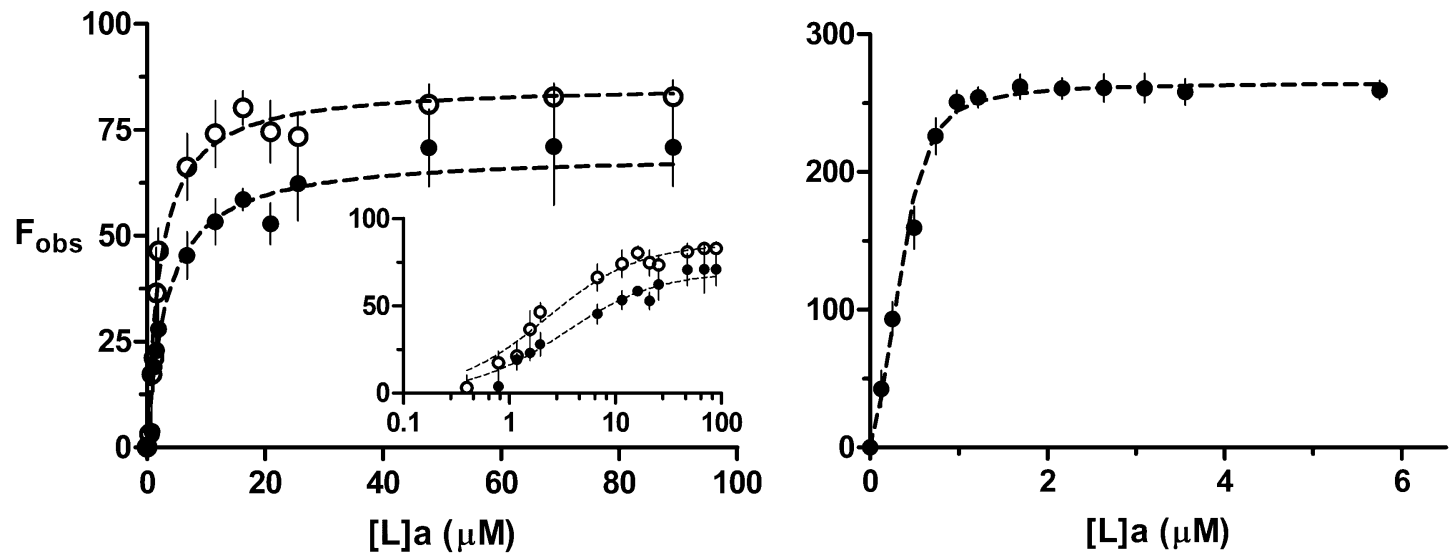

Figure 1. Binding curve for a $\mathrm{Ni}^{2+} \rightarrow C j$ NikZ titration (left-panel) in $20 \mathrm{mM}$ MOPS pH 8 (empty circles) and $40 \mathrm{mM}$ HEPES pH 7.5 (filled circles), as well as a (1:2)Ni-His $\rightarrow$ CjNikZ titration (right-panel) in $40 \mathrm{mM} \mathrm{HEPES} \mathrm{pH} \mathrm{7.5.} \mathrm{Curves} \mathrm{of}$ best-fit are generated with Equation (7) on Prism (left-panel), and the KK method with Equation (6) and $n$ on Excel (right-panel). An x-axis logarithmic plot of $\mathrm{Ni}^{2+} \rightarrow C$ CNikZ is provided for closer visual inspection (left-panel inset).

at the expense flattening the curve for $F_{\max }$ to the point where a reasonable $\mathrm{Cl}$ could not be calculated, which is symptomatic of overparameterization of the model by $n .{ }^{36}$ Overall, this indicates $n$ should be used judiciously, and only when minimizations of $\chi^{2}$ are substantial and biologically meaningful.

In Lebrette et al. (2014), they complement their ITC data for CjNikZ with three crystal structures, one of which includes $\mathrm{CjNikZ}$ bound to $\mathrm{Ni}^{2+}$ chelated by a histidine (PDB ID: 4OEU). Upon closer analysis, a (very) well-formed octahedral coordination complex is created between CjNikZ, $\mathrm{Ni}^{2+}$, and histidine. However, their ITC data revealed that CjNikZ did not produce any thermic event when $\mathrm{Ni}(\mathrm{His})_{2}$ was injected. While they explain the histidine in the crystal structure was an artifact of their crystallization procedure, we performed a Ni(His) ${ }_{2} \rightarrow C j N i k Z$ microlTFQ-LTA in $40 \mathrm{mM}$ HEPES pH 7.5, the same buffer 
conditions for the ITC they performed, and detected a low nanomolar binding event. Using the KK method with Equation (6) and inclusion of $\mathrm{n}$, we observed a strong binding event: $\mathrm{n}$ of 1.32 with a $\mathrm{Cl}$ of 1.11 to $1.57, K_{\mathrm{D}}$ of $51 \mathrm{nM}$ (!) with a $\mathrm{Cl}$ of 31 to $74 \mathrm{nM}$, and an $F_{\max }$ of 265.25 with a $\mathrm{Cl}$ of 255.23 to 278.65 (Table 1). Of pertinence, we subtracted the background fluorescence emission of histidine at each titration step through an additional set of controls to remove the IFE. Nonetheless, the IFE contribution of histidine only began appearing in the last two data points reported in Figure 1. We were not surprised by this finding because $\mathrm{Ni}$-BPs have been found to bind to $\mathrm{Ni}$-His complexes before with nanomolar affinity, which includes other structures solved in the same study by Lebrette et al. (2014). It is possible that in their study a small amount of $\mathrm{Ni}(\mathrm{His})_{2}$ was accidentally exposed to $\mathrm{CjNikZ}_{\mathrm{N}}$ in the sample cell upon insertion of the syringe into the sample, or by some other means, which would have saturated the protein prior to the first injection.

Compared to the usage of $\mathrm{n}$ for $\mathrm{Ni}^{2+} \rightarrow C j \mathrm{NikZ}$ in $40 \mathrm{mM}$ HEPES pH 7.5 where it had a detrimental role on computing $\mathrm{Cls}$ by the KK method, $\mathrm{n}$ plays an important role in properly fitting Equations (6) and (7) to the data here. This is illustrated in Supplementary Figure 6 where the curves for both $K_{\mathrm{D}}$ and $F_{\max }$ were lowered and slightly narrowed by unconstraining $\mathrm{n}$ (viz. including it in the model), and Supplementary Figure 7 where the curve for $\mathrm{n}$ was lowered and noticeably narrowed when applied to

Table 1. Binding parameters for CjNiKZ

\begin{tabular}{|c|c|c|c|c|c|c|c|c|c|c|c|}
\hline \multicolumn{12}{|c|}{$\mathrm{Ni} \rightarrow$ CjNiKZ, 40 mM HEPES pH 7.5} \\
\hline \multirow{2}{*}{$\begin{array}{c}\text { NLLS } \\
\text { Method }\end{array}$} & \multirow{2}{*}{ Model } & \multirow{2}{*}{$\chi^{2}$} & \multirow{2}{*}{$\mathbf{n}$} & \multicolumn{2}{|c|}{$\mathrm{n}, 95 \% \mathrm{Cl}$} & \multirow{2}{*}{$\begin{array}{c}\boldsymbol{K}_{\mathbf{D}} \\
(\mu \mathrm{M})\end{array}$} & \multicolumn{2}{|c|}{$\mathrm{k}_{\mathrm{D}}, 95 \% \mathrm{Cl}$} & \multirow{2}{*}{$F_{\max }$} & \multicolumn{2}{|c|}{$\mathrm{F}_{\max }, 95 \% \mathrm{Cl}$} \\
\hline & & & & Lower & Upper & & Lower & Upper & & Lower & Upper \\
\hline \multirow{2}{*}{ Prism } & \multirow{2}{*}{ Eq.(7) } & 4.45 & 1 & & & 3.34 & 2.41 & 4.26 & 69.40 & 64.64 & 74.17 \\
\hline & & 4.32 & 0.90 & 0.60 & 1.21 & 3.39 & 2.39 & 4.39 & 72.95 & 58.93 & 86.96 \\
\hline \multirow{2}{*}{ KK } & \multirow{2}{*}{$\mathrm{Eq} \cdot(7)$} & 4.45 & 1 & & & 3.34 & 2.57 & 4.27 & 69.41 & 65.32 & 74.14 \\
\hline & & 4.31 & 0.91 & 0.67 & 1.26 & 3.39 & 2.63 & 4.41 & 72.94 & \multicolumn{2}{|c|}{ Error $^{a}$} \\
\hline \multirow{2}{*}{ KK } & \multirow{2}{*}{ Eq.(6) } & 4.56 & 1 & & & 3.02 & 2.24 & 3.92 & 68.59 & 64.49 & 73.37 \\
\hline & & 4.32 & 0.88 & 0.65 & 1.23 & 3.08 & 2.34 & 4.10 & 73.03 & \multicolumn{2}{|c|}{ Error ${ }^{a}$} \\
\hline
\end{tabular}

$(1: 2)^{\mathrm{b}} \mathrm{Ni}-\mathrm{His} \rightarrow \mathrm{CjNiKZ}, 40 \mathrm{mM}$ HEPES pH 7.5

\begin{tabular}{|c|c|c|c|c|c|c|c|c|c|c|c|}
\hline \multirow{2}{*}{$\begin{array}{c}\text { NLLS } \\
\text { Method }\end{array}$} & \multirow{2}{*}{ Model } & \multirow{2}{*}{$x^{2}$} & \multirow{2}{*}{$\mathbf{n}$} & \multicolumn{2}{|c|}{$\mathrm{n}, 95 \% \mathrm{Cl}$} & \multirow{2}{*}{$\begin{array}{c}\boldsymbol{K}_{\mathrm{D}} \\
(\mu \mathrm{M})\end{array}$} & \multicolumn{2}{|c|}{ kD, 95\% Cl } & \multirow{2}{*}{$F_{\max }$} & \multicolumn{2}{|c|}{$\mathrm{F}_{\max }, 95 \% \mathrm{Cl}$} \\
\hline & & & & Lower & Upper & & Lower & Upper & & Lower & Upper \\
\hline \multirow{2}{*}{ Prism } & \multirow{2}{*}{ Eq.(7) } & 53.17 & $1^{\mathrm{a}}$ & & & 0.30 & 0.13 & 0.47 & 293 & 265.20 & 320.80 \\
\hline & & 6.74 & 2.17 & 1.71 & 2.63 & 0.090 & 0.038 & 0.14 & 264.7 & 256.90 & 272.50 \\
\hline \multirow{2}{*}{ KK } & \multirow{2}{*}{ Eq.(7) } & 53.17 & $1^{a}$ & & & 0.30 & 0.19 & 0.45 & 293.02 & 267.65 & 328.05 \\
\hline & & 6.74 & 2.17 & 1.83 & 2.58 & 0.090 & 0.056 & 0.13 & 264.69 & 258.70 & 271.24 \\
\hline \multirow{2}{*}{ KK } & \multirow{2}{*}{ Eq.(6) } & 16.38 & $1^{\mathrm{a}}$ & & & 0.093 & 0.050 & 0.15 & 272.36 & 261.22 & 284.48 \\
\hline & & 4.42 & 1.32 & 1.11 & 1.57 & 0.051 & 0.031 & 0.074 & 265.25 & 255.23 & 278.65 \\
\hline
\end{tabular}

a. $\mathrm{Cl}$ could not be computed due to an inability to define a local minimum (refer to Supplementary Figures 5 ) b. a 1:2 stochiometric ligand solution of $\mathrm{NiSO}_{4}$ and L-histidine

310 Equation (6). By including $n, \chi^{2}$ was reduced by $88 \%$ and $73 \%$ for Equation (7) and (6), respectively. Overall, 311 this provides evidence supporting the functional role of the histidine in assisting $C j \mathrm{NikZ}$ in binding $\mathrm{Ni}^{2+}$ 312 more tightly and helps explain why histidine is found inside the crystal structure of CjNikZ. Next, we 313 demonstrate the microlTFQ-LTA's utility in understanding how CCSBPII binds to various metal ligands under different conditions. The best-fit parameter values are summarized in Table 2. 
Table 2. Binding parameters for CCSBPII

\begin{tabular}{|c|c|c|c|c|c|c|c|c|c|}
\hline \multirow{2}{*}{ Buffer ${ }^{a}$} & \multirow{2}{*}{$\mathrm{n}$} & \multicolumn{2}{|c|}{$\mathrm{n}, \mathbf{9 5 \%} \mathrm{Cl}$} & \multirow{2}{*}{$\begin{array}{c}K_{\mathrm{D}} \\
(\mu \mathrm{M})\end{array}$} & \multicolumn{2}{|c|}{$K_{\mathrm{D}}, 95 \% \mathrm{Cl}$} & \multirow{2}{*}{$F_{\max }$} & \multicolumn{2}{|c|}{$F_{\max }, 95 \% \mathrm{Cl}$} \\
\hline & & Lower & Upper & & Lower & Upper & & Lower & Upper \\
\hline 10 mM HEPES pH 7.2 & & & & 13.40 & 10.71 & 16.09 & 178.42 & 170.22 & 186.63 \\
\hline 10 mM MOPS pH 7.2 & & & & 25.83 & 19.68 & 31.98 & 196.89 & 185.51 & 208.27 \\
\hline 10 mM PIPES pH 7.2 & & & & 22.08 & 17.66 & 26.50 & 185.58 & 175.99 & 195.17 \\
\hline $10 \mathrm{mM}$ Tris $\mathrm{pH} 7.2$ & & & & 16.18 & 13.90 & 18.47 & 169.33 & 163.05 & 175.60 \\
\hline 10 mM Phosphate $\mathrm{pH} 7.2$ & & & & \multicolumn{6}{|c|}{ Error ${ }^{b-1}$} \\
\hline 10 mM HEPES $\mathrm{pH} 7.2$ & & & & 12.67 & 10.77 & 14.56 & 174.90 & 167.61 & 182.18 \\
\hline 20 mM HEPES pH 7.2 & & & & 12.24 & 10.19 & 14.29 & 168.05 & 163.10 & 172.99 \\
\hline 40 mM HEPES pH 7.2 & & & & 15.85 & 12.74 & 18.96 & 160.09 & 152.90 & 167.28 \\
\hline 100 mM HEPES pH 7.2 & & & & 30.76 & 22.78 & 38.75 & 152.81 & 142.81 & 162.80 \\
\hline $1000 \mathrm{mM}$ HEPES $\mathrm{pH} 7.2$ & & & & 19.36 & 4.81 & 33.92 & 23.38 & 18.57 & 28.18 \\
\hline $10 \mathrm{mM}$ HEPES $0 \mathrm{mM} \mathrm{NaCl}, \mathrm{pH} 7.2$ & & & & 13.75 & 11.52 & 15.98 & 185.31 & 177.85 & 192.77 \\
\hline $10 \mathrm{mM}$ HEPES $10 \mathrm{mM} \mathrm{NaCl}, \mathrm{pH} 7.2$ & & & & 18.47 & 15.03 & 21.92 & 181.64 & 173.42 & 189.86 \\
\hline $10 \mathrm{mM}$ HEPES $100 \mathrm{mM} \mathrm{NaCl}, \mathrm{pH} 7.2$ & & & & 46.65 & 37.60 & 55.70 & 172.18 & 161.62 & 182.75 \\
\hline 10 mM HEPES 1000 mM NaCl, pH 7.2 & & & & 77.35 & 34.15 & 120.54 & 143.16 & 96.54 & 189.78 \\
\hline 10 mM HEPES pH 2.5 & & & & \multicolumn{6}{|c|}{ Error b-2 } \\
\hline 10 mM HEPES pH 6.5 & & & & 39.24 & 32.68 & 45.79 & 142.87 & 136.65 & 149.08 \\
\hline 10 mM HEPES pH 7.0 & & & & 22.34 & 18.63 & 26.04 & 155.04 & 147.61 & 162.48 \\
\hline 10 mM HEPES pH 7.2 & & & & 13.76 & 11.59 & 15.92 & 175.61 & 167.44 & 183.77 \\
\hline 10 mM HEPES $\mathrm{pH} 7.5$ & & & & 12.31 & 10.20 & 14.42 & 171.84 & 165.46 & 178.22 \\
\hline $10 \mathrm{mM}$ HEPES $\mathrm{pH} 8.0$ & & & & 5.39 & 3.89 & 6.88 & 204.48 & 196.83 & 212.12 \\
\hline \multirow{2}{*}{ Ligand $^{c}$} & \multirow{2}{*}{$\mathrm{n}$} & \multicolumn{2}{|c|}{$\mathrm{n}, \mathbf{9 5 \%} \mathrm{Cl}$} & \multirow{2}{*}{$\begin{array}{c}K_{\mathrm{D}} \\
(\mu \mathrm{M})\end{array}$} & \multicolumn{2}{|c|}{$K_{\mathrm{D}}, \mathbf{9 5 \%} \mathrm{Cl}$} & \multirow{2}{*}{$F_{\max }$} & \multicolumn{2}{|c|}{$F_{\max }, 95 \% \mathrm{Cl}$} \\
\hline & & Lower & Upper & & Lower & Upper & & Lower & Upper \\
\hline $\mathrm{NiSO}_{4}$ & & & & 14.13 & 13.27 & 14.98 & 182.51 & 178.74 & 186.28 \\
\hline $\mathrm{NiCl}_{2}$ & & & & 12.00 & 11.12 & 12.88 & 176.68 & 172.70 & 180.66 \\
\hline $\mathrm{CoCl}_{2}$ & & & & 21.11 & 13.62 & 28.60 & 95.99 & 86.11 & 105.87 \\
\hline $\mathrm{CuCl}_{2}$ & & & & 8.54 & 5.81 & 11.28 & 595.10 & 552.82 & 637.38 \\
\hline $\mathrm{MnCl}_{2}$ & & & & 98.00 & 83.00 & 113.01 & 104.10 & 98.39 & 109.80 \\
\hline $\mathrm{ZnCl}_{2}$ & & & & 153.89 & 85.88 & 221.90 & 236.20 & 211.06 & 261.35 \\
\hline $\mathrm{CdCl}_{2}$ & & & & $11.61^{d}$ & 8.70 & 14.52 & 259.93 & 240.40 & 279.47 \\
\hline$(1: 2) \mathrm{Ni}-\mathrm{His}^{f}$ & 0.83 & 0.80 & 0.86 & $4.40^{e}$ & 1.33 & 8.76 & 350.42 & 347.22 & 354.06 \\
\hline$(1: 2)$ Co-His $^{f}$ & 1.21 & 1.13 & 1.29 & $557.85^{\mathrm{e}}$ & 509.88 & 608.20 & 439.38 & 430.69 & 448.83 \\
\hline$(1: 2) \mathrm{Cu}-\mathrm{His}^{f}$ & 1.19 & 1.02 & 1.38 & $36.08^{e}$ & 14.69 & 65.45 & 425.31 & 414.08 & 437.36 \\
\hline$(1: 2) \mathrm{Ni}-\mathrm{Imi}^{f}$ & 0.82 & 0.77 & 0.88 & 6.76 & 6.00 & 7.51 & 222.32 & 216.74 & 227.90 \\
\hline$(1: 2)$ Ni-Gly ${ }^{f}$ & 0.80 & 0.70 & 0.90 & 13.00 & 9.89 & 16.10 & 348.06 & 335.77 & 360.35 \\
\hline$(1: 2: 2)$ Ni-Imi-Gly ${ }^{f}$ & 0.86 & 0.74 & 0.98 & 14.35 & 10.37 & 18.32 & 367.10 & 343.56 & 390.64 \\
\hline
\end{tabular}

Best-fit parameter values and 95\% confidence intervals $(\mathrm{Cl})$ are by default calculated on Prism without inclusion of $\mathrm{n}$, meaning that $\mathrm{n}=1$, and using Equation (7) unless otherwise indicated.

a. ligand used is $\mathrm{Ni}^{2+}$ prepared with $\mathrm{NiCl}_{2}$

b-1. saturation was not achieved in the ligand range titrated

b-2. no change in fluorescence occurred compared to the controls

c. 10 mM HEPES pH 7.2 is used in all cases for CCSBPII and to prepare the ligand solutions

d. values and corresponding lower and upper bound for $\mathrm{Cl}$ is reported in millimolar (mM)

e. values and corresponding lower and upper bound for $\mathrm{Cl}$ is reported in nanomolar (nM)

$f$. the KK method with inclusion of $n$, meaning $n$ is unconstrained, is used with Equation (6) instead His: L-histidine, Gly: L-glycine, Imi: imidazole 
It is well known that $\mathrm{pH}$ buffers are crucial for numerous biochemical, cellular, and environmental assays; it is also known that buffer molecules can interact with the sample and confound the analysis, which is especially true for metal-binding studies. We performed $\left(\mathrm{NiCl}_{2}\right) \mathrm{Ni}^{2+} \rightarrow$ CCSBPII microlTFQ-LTAs with various Good's buffers claimed to be "metal-compatible" buffers ${ }^{37}$, as well as phosphate buffer (Figure 2). Using Equation (7) in Prism with $n=1,10 \mathrm{mM}$ HEPES, PIPES, MOPS, and Tris at pH 7.2 all generated similar binding curves with $K_{\mathrm{D}}$ values ranging from 13 to $25 \mu \mathrm{M}$ (Table 2). These buffers all possess lone pair electron orbitals at $\mathrm{pH} 7.2$ and can therefore act as Lewis bases to form coordinate bonds with $\mathrm{Ni}^{2+}{ }^{38}$ While this may explain the minor differences in $K_{\mathrm{D}}$ between the four buffers, their Cls show some overlap. This is also true for the $F_{\max }$ values and their Cls. It is likely that the microlTFQ-LTAs could not distinguish between the effects the four buffers had on $\mathrm{Ni}^{2+} \rightarrow C c S B P I I$, which indicated to us that the
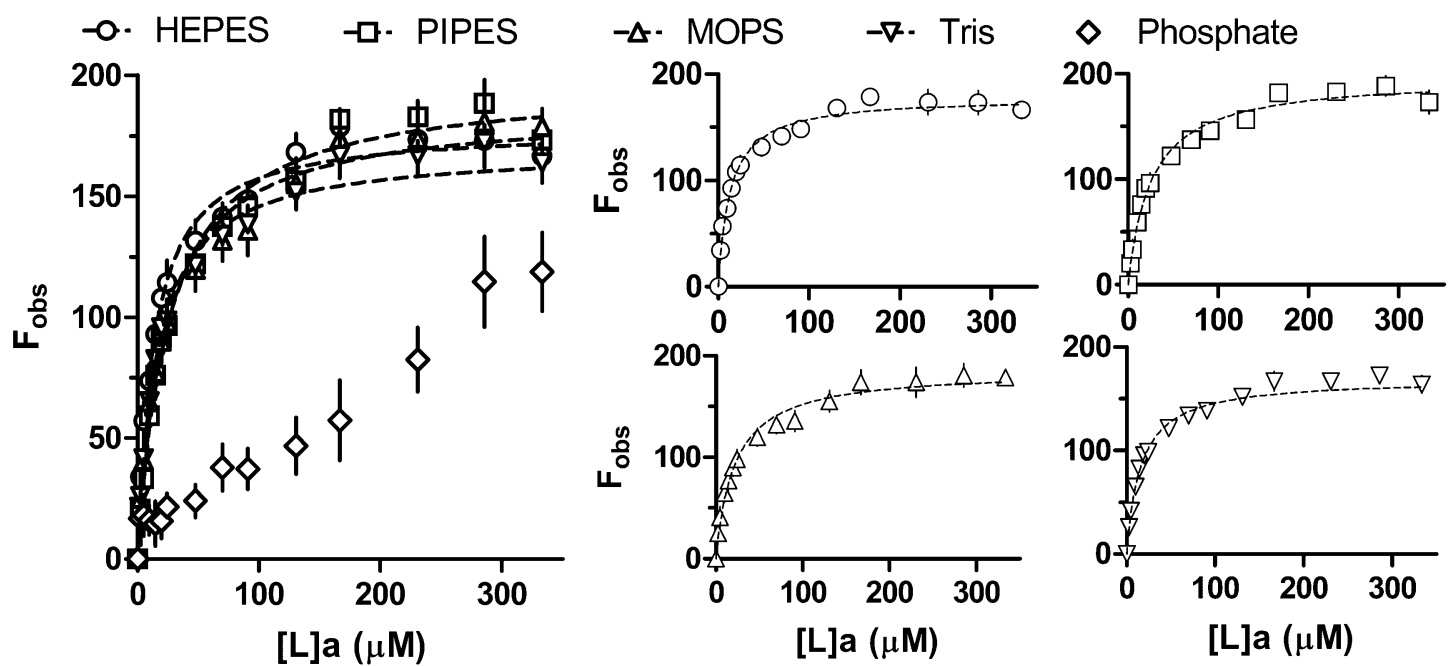

Figure 2. Binding curves for $\mathrm{Ni}^{2+} \rightarrow C c$ SBPII titrations (left-panel) at $\mathrm{pH} 7.2$ for $10 \mathrm{mM}$ HEPES (circle), $10 \mathrm{mM}$ PIPES (square), $10 \mathrm{mM}$ MOPS (upward triangle), $10 \mathrm{mM}$ Tris (downward triangle), and $10 \mathrm{mM}$ phosphate (diamond). Right panels show the same curves in the left panel individually (except $10 \mathrm{mM}$ phosphate) for easier visual inspection.
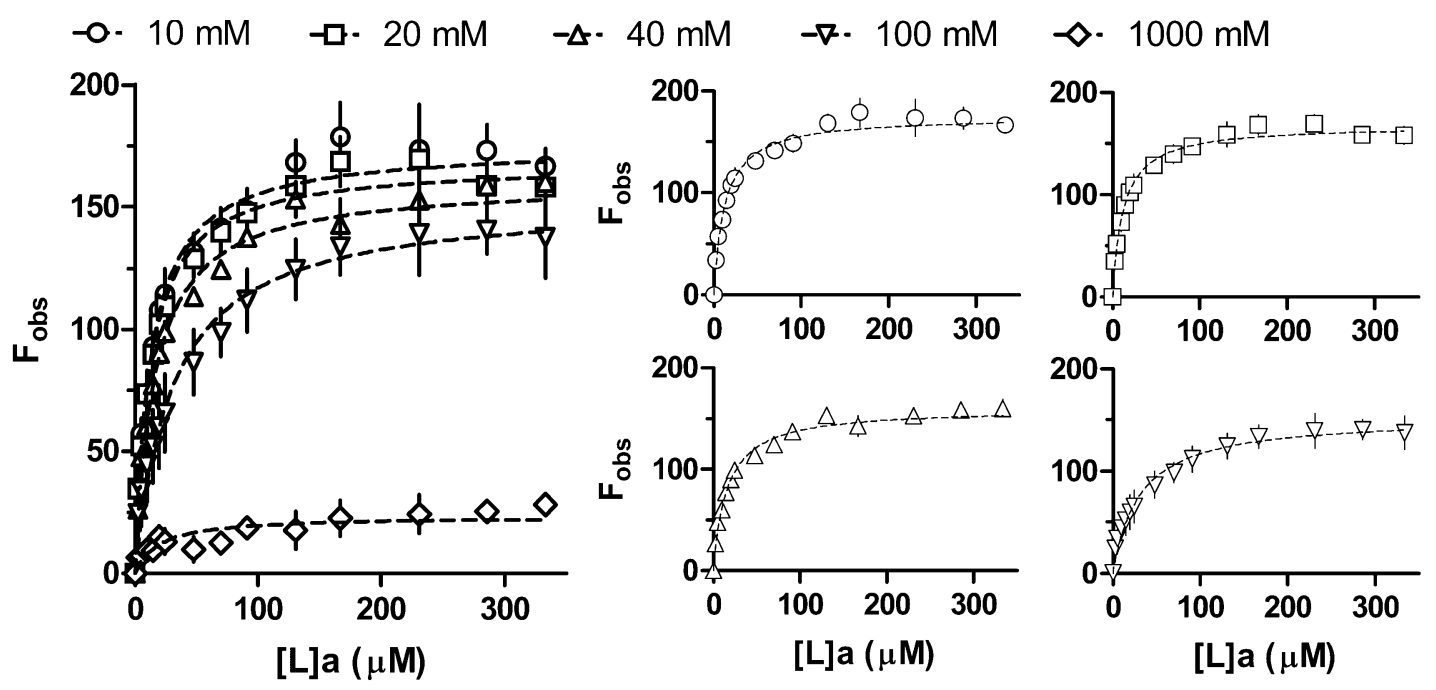

Figure 3. Binding curves for $\mathrm{Ni}^{2+} \rightarrow C c S B P I$ titrations (left-panel) at $\mathrm{pH} 7.2$ with HEPES at $10 \mathrm{mM}$ (circle), $20 \mathrm{mM}$ (square), $40 \mathrm{mM}$ (upward triangle), $100 \mathrm{mM}$ (downward triangle), and $1000 \mathrm{mM}$ (diamond). Right panels show the same curves in the left panel individually (except $1000 \mathrm{mM}$ HEPES) for easier visual inspection. 
use of any of these four buffers is appropriate since saturation could be achieved, as long the selected buffer is consistently used in further assays. A binding curve could not be generated with the phosphate buffered likely because metal-phosphate complexes tend to precipitate, thus preventing CCSBPII from interacting with the metal. ${ }^{39}$ Therefore, we continued testing HEPES as the buffer of choice because it provided the lowest $K_{\mathrm{D}}$, suggesting the buffer interfered the least with the $\mathrm{Ni}^{2+} \rightarrow C C S B P I I$ interaction.

When varying [HEPES] from $10-1000 \mathrm{mM}$ at $\mathrm{pH} 7.2$ and using Equation (7) in Prism with $\mathrm{n}=1$, we found the binding curves changed in two ways (Figure 3 ). $K_{\mathrm{D}}$ increased up to 2.4 -fold over a change in 1-2 orders of magnitude of [HEPES], possibly because the higher concentrations of HEPES molecules effectively siphoned $\mathrm{Ni}^{2+}$ from the pool available for CCSBPII to bind to, meaning that $[L]_{\text {free }}$ for each titration step was overestimated and lead to a rightward-positive shift of the binding curve. More notably, $F_{\max }$ decreased 7.5-fold over a change in 2 orders of magnitude of [HEPES]. Specifically $F_{0}$, which is needed to calculate $F_{\max }$, decreased and indicates the fluorescence from tryptophans had already been reduced
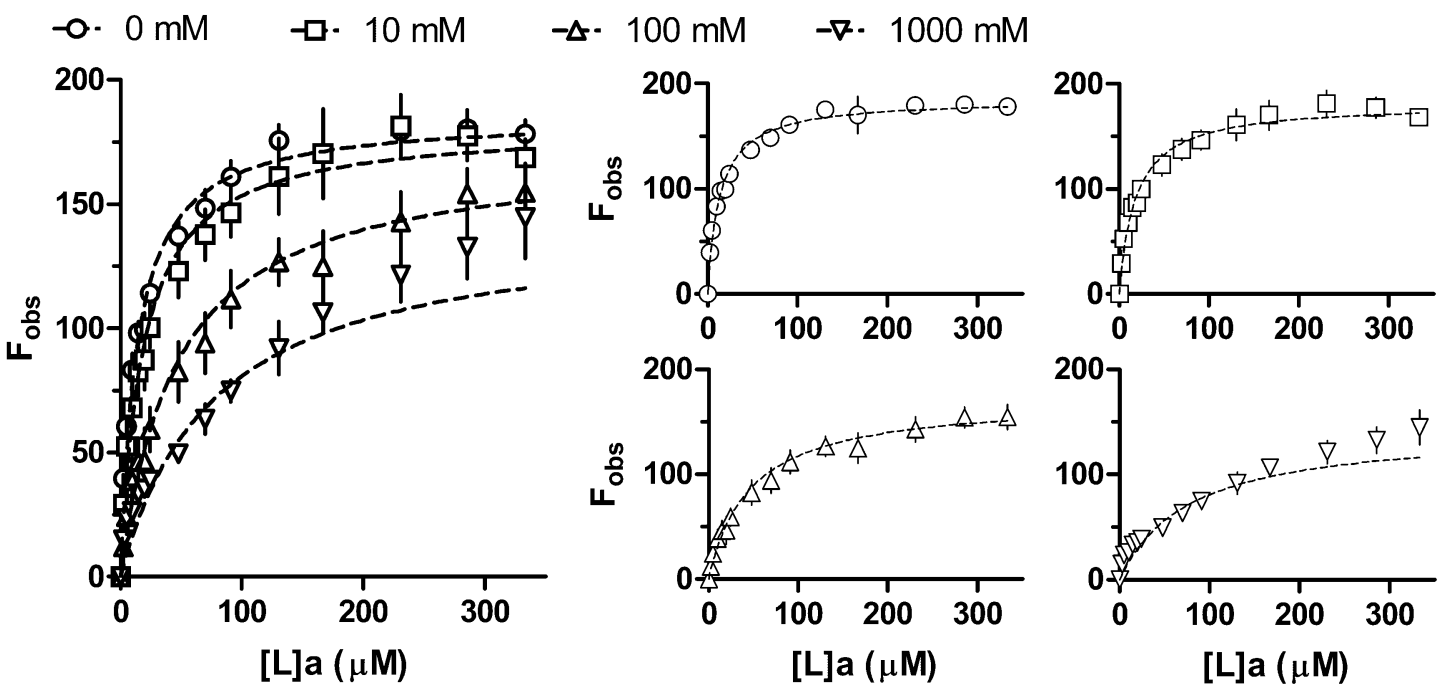

Figure 4. Binding curves for $\mathrm{Ni}^{2+} \rightarrow C c S B P I I$ titrations (left-panel) at $\mathrm{pH} 7.2$ with $10 \mathrm{mM} \mathrm{HEPES}$ and $\mathrm{NaCl}$ at $0 \mathrm{mM}$ (circle), $10 \mathrm{mM}$ (square), $100 \mathrm{mM}$ (upward triangle), and $1000 \mathrm{mM}$ (downward triangle). Right panels show the same curves in the left panel individually (except pH 2.5 and pH 8.0) for easier visual inspection.

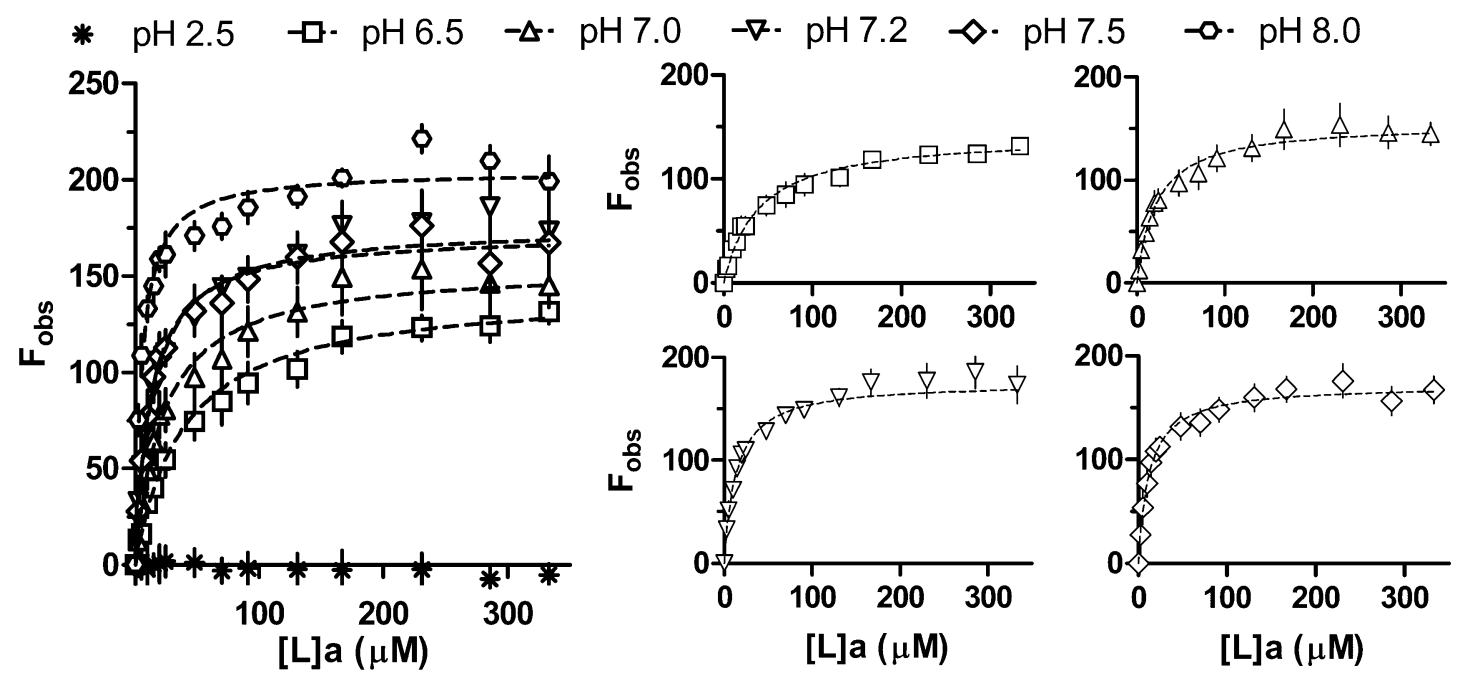

Figure 5. Binding curves for $\mathrm{Ni}^{2+} \rightarrow C c S B P I I$ titrations (left-panel) with $10 \mathrm{mM}$ HEPES at pH 2.5 (asterisk), pH 6.5 (square), pH 7.0 (upward triangle), pH 7.2 (downward triangle), pH 7.5 (diamond), and pH 8 (hexagon). Right panels show the same curves in the left panel individually (except $\mathrm{pH} 2.5$ and $\mathrm{pH} 8.0$ ) for easier visual inspection. 
prior to the first titration, noting here that $1000 \mathrm{mM}$ HEPES displayed a negligible increase in absorbance at the wavelengths used compared to $10 \mathrm{mM}$ HEPES (data not shown). At higher concentrations, it is likely that HEPES exerts a chaotropic effect on the CCSBPII's hydration shell, thus partially denaturing CcSBPII. This could alter the environments surrounding the tryptophans as well as the binding cavity, which could also explain why $K_{\mathrm{D}}$ appeared to increase. ${ }^{40,41}$ Circular dichroism, thermal shift assays, or any method to probe changes in the secondary structure are needed to explore this. However, [HEPES] in the $1000 \mathrm{mM}$ range is not typical of or practical for biochemical experiments and it was simpler to choose a lower [HEPES] with sufficient $\mathrm{pH}$ buffering capacity for the microITFQ-LTAs, so we continued with $10 \mathrm{mM}$ HEPES.

When varying [ $\mathrm{NaCl}$ from 0 to $1000 \mathrm{mM}$ at pH 7.2, and using Equation (7) in Prism with $\mathrm{n}=1$, we found the binding curves changed in a similar manner to when [HEPES] was varied (Figure 4). $K_{\mathrm{D}}$ increased 5.6-fold and $F_{\text {max }}$ decreased 1.3-fold from 0 to $1000 \mathrm{mM} \mathrm{NaCl}$, which likely reflects chaotropic effects on CCSBPII's hydration shell exerted by high $[\mathrm{NaCl}]$. C. carboxidvorans is not known to be a halophile, meaning that its proteins are unlikely to form salt-bridge networks on their surfaces that would otherwise stabilize the structure. The more pronounced effect on $K_{\mathrm{D}}$ compared to the case with high [HEPES] may be due to $\mathrm{Na}^{+}$. While it is a hard ion that unlikely competes directly with $\mathrm{Ni}^{2+}$ to form coordinate bonds with CcSBPII's binding residues, its positive charge could still lead to electrostatic interactions with the negativelycharged binding cavity (Supplementary Figure 8), thus hindering the entrance and binding of $\mathrm{Ni}^{2+}$. Therefore, we chose not to include $[\mathrm{NaCl}]$ to minimize the number of buffer components that could complicate the $\mathrm{Ni}^{2+} \rightarrow C C S B P I I$ interaction and continued with $10 \mathrm{mM}$ HEPES.

When varying the $\mathrm{pH}$ from 6.5 to 8.0 for $10 \mathrm{mM}$ HEPES, and using Equation (7) in Prism with $\mathrm{n}=$ 1 , we found $K_{\mathrm{D}}$ decreased 7.3 -fold and $F_{\text {max }}$ increased 1.4 -fold in contrast to increasing [HEPES] and [NaCl], indicating a stronger binding event in more alkaline conditions (Figure 5). No change in fluorescence was observed at $\mathrm{pH} 2.5$. Generally, $\mathrm{pH}$ plays a crucial role in metalloprotein function because it affects the ionization of the sidechains involved in binding to metal ions. ${ }^{40,42}$ Two of the four hypothesized binding residues based on structural superimposition with CjNikZ (Supplementary Figure 2) are histidines that have a $\mathrm{pK}_{\mathrm{a}}$ of 6.0 for the imidazole side chain; a $\mathrm{pH}$ below this value would protonate their nitrogens rendering them unable to contribute the lone pairs of electrons to form coordinate bonds with $\mathrm{Ni}^{2}$. With increasing $\mathrm{pH}$ from 6.5 to 8.0 , these histidines could become increasingly deprotonated such that coordinate bonds can be formed more easily with $\mathrm{Ni}^{2+}$. The other two hypothesized binding residues are lysine ( $\mathrm{pK}_{\mathrm{a}}$ 10.54) and arginine $\left(\mathrm{pK}_{\mathrm{a}} 12.48\right)$, which may experience some deprotonation in the $\mathrm{pH}$ range tested that would allow for better coordinate bond formation, though this effect is likely minor due to their large $\mathrm{pK}_{\mathrm{a}}$ values. To avoid CCSBPII's isoelectric point ( $\mathrm{pl} 5.78$ ) and to limit nickel hydroxide precipitation beginning at $\mathrm{pH} 8.5$ to $9,{ }^{43}$ we defined $10 \mathrm{mM} \mathrm{HEPES}$ at $\mathrm{pH} 7.2$ as a suitable buffer condition to conduct further ligand screening for CCSBPII.

\section{microlTFQ-LTA for ligand screening with CCSBPII in a defined buffer}

High-throughput ligand screens using thermal shift assays for SBPs have been conducted for metabolic intermediates, carbohydrates, amino acids, and sulfur compounds. ${ }^{4-46}$ In these screens, SBPs were tested for binding to myriad compounds. In contrast, Ni-BPs are often assumed to be isofunctional for a single metal ion and their promiscuity with other metal ions rarely ever reported. In our analysis of the peptide/nickel binding protein family (InterPro ID: IPR0306078), CCSBPII clustered in the same phylogenetic branch as CjNikZ and nearby other characterized Ni-BPs (data not shown). Based on this information and the structural superimposition of CCSBPII to CjNikZ (Supplementary Figure 2), we hypothesized CCSBPII's target metal ion is $\mathrm{Ni}^{2+}$. In the previous section, we provided evidence for a $\mathrm{Ni}^{2+} \rightarrow$ CcSBPII binding event with low micromolar affinity in $10 \mathrm{mM} \mathrm{HEPES} \mathrm{pH} \mathrm{7.2.} \mathrm{Metal} \mathrm{SBPs} \mathrm{with} \mathrm{crystal}$ structures on the Protein Data Bank (PDB) have been solved with $\mathrm{Co}^{2+}, \mathrm{Cu}^{2+}, \mathrm{Zn}^{2+}, \mathrm{Mn}^{2+}$, or $\mathrm{Cd}^{2+}$ inside the binding cavity. Therefore, we performed a small ligand screen for CCSBPII's interactions with these other 
metal ions in $10 \mathrm{mM} \mathrm{HEPES} \mathrm{pH} 7.2$ (Figure 6). When $\mathrm{NiCl}_{2}$ and $\mathrm{NiSO}_{4}$ were used to prepare the $\mathrm{Ni}^{2+}$ ligand solution, similar $K_{\mathrm{D}}$ values were obtained for CCSBPII: $14.13 \mu \mathrm{M}$ with a $\mathrm{Cl}$ of 13.27 to $14.98 \mu \mathrm{M}$, and 12.00 $\mu \mathrm{M}$ with a $\mathrm{Cl}$ of 11.12 to $12.88 \mu \mathrm{M}$, respectively. Since they are nearly identical, and because we previously used $\mathrm{NiCl}_{2}$, we prepared the other metal ligands using their chloride salt to be consistent.

The results are summarized in Table 2 . Binding affinities $\left(K_{\mathrm{D}}\right)$ varied over 3 orders of magnitude, and the observed order of weakest to strongest affinity was $\mathrm{Cd}^{2+}<<\mathrm{Zn}^{2+}<\mathrm{Mn}^{2+}<\mathrm{Co}^{2+}<\mathrm{Ni}^{2+}<\mathrm{Cu}^{2+}$. Notably, $\mathrm{Cd}^{2+}$ and $\mathrm{Zn}^{2+}$ are spectroscopically silent metal ions due to their complete $d$ orbitals, yet they still generated a saturable signal in the microlTFQ-LTA. This indicates the mechanism of fluorescence quenching was not collisional quenching, but better explained by the changes in the polarity of the environment surrounding the proteinaceous tryptophans due to binding-induced conformational changes. This is confirmed by other binding studies using specITFQ-LTA to study $\mathrm{Cd}^{2+}$ and $\mathrm{Zn}^{2+}$ interactions with metalloproteins. ${ }^{47,48}$

Also of note, the observed order resembles the natural order of stabilities (i.e. the Irving-William series). ${ }^{49,50}$ However, an anomaly in this observed order is $\mathrm{Zn}^{2+}$ appearing to be weaker in affinity than $\mathrm{Co}^{2+}$, whereas in the originally reported Irving-William series $\mathrm{Zn}^{2+}$ is predicted to have a stronger affinity than $\mathrm{Co}^{2+}$. The theoretical and empirical rationale for the order of the Irving-William series is based on observations made with mono- to poly-dentate amino-bearing molecules in aqueous solution, and highspin octahedral complexes..$^{50}$ While amino acid monomers were considered in this conceptualization, it
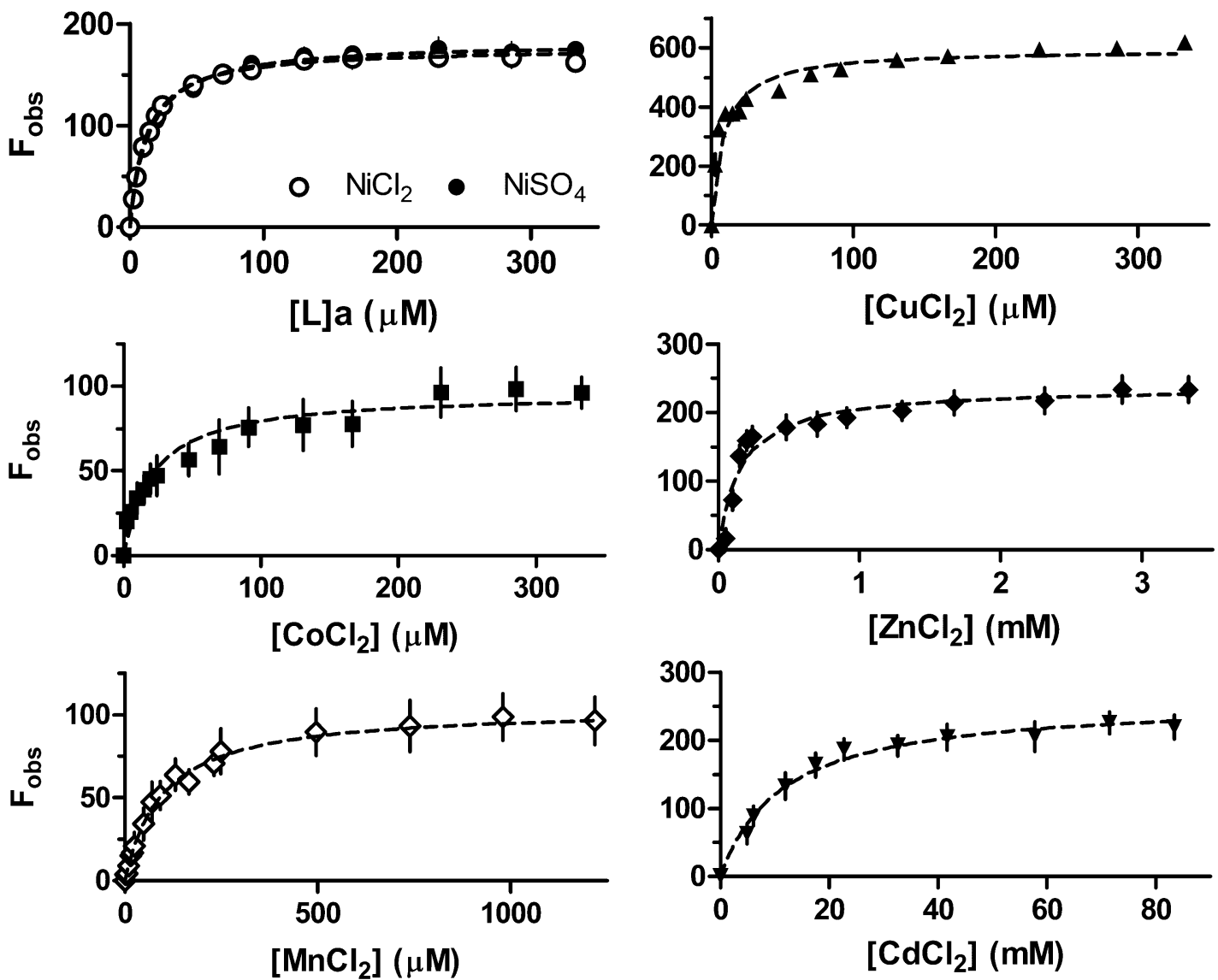

Figure 6. Binding curves for $\mathrm{M}^{2+} \rightarrow$ CCSBPII titrations in $10 \mathrm{mM} \mathrm{HEPES} \mathrm{pH} 7.2$, where $\mathrm{M}$ represents a divalent metal: $\mathrm{NiCl}_{2}$ (empty circle), $\mathrm{NiSO}_{4}$ (filled circle), $\mathrm{CuCl}_{2}$ (upward triangle), $\mathrm{CoCl}_{2}$ (square), $\mathrm{ZnCl}_{2}$ (filled diamond), $\mathrm{MnCl}_{2}$ (empty diamond), $\mathrm{CdCl}_{2}$ (downward triangle). Metal ligands are prepared in $10 \mathrm{mM} \mathrm{HEPES} \mathrm{pH} 7.2 \mathrm{Note}$ the differences in range for the $y$-axis and $x$-axis when comparing curves. 
did not consider the spatial constraints imparted by a relatively rigid protein structure that may only be able to accommodate a specific coordination geometry. $\mathrm{Zn}^{2+}$ is overwhelmingly found in metalloproteins with a tetrahedral geometry despite its tendency to form an octahedral $\left[\mathrm{Zn}\left(\mathrm{H}_{2} \mathrm{O}\right)_{6}\right]^{2+}$ complex in an aqueous solution. ${ }^{51,52}$ It is possible that CCSBPII's hypothesized binding residues are unable to readily position themselves to accommodate a ligand with a tetrahedral preference, perhaps requiring a conformational change to do so that would explain its 1.47-fold increase in $\mathrm{F}_{\max }$ compared to $\mathrm{Ni}^{2+}$. $\mathrm{Zn}^{2+}$ also has a stronger preference for softer elements like sulfur in cysteine, of which is not found in the CCSBPII binding cavity.

For $\mathrm{Cd}^{2+} \rightarrow C C S B P I I$, it is unclear why exactly its $K_{\mathrm{D}}$ increased over 967.5 -fold compared to $\mathrm{Ni}^{2+}$ when $\mathrm{Zn}^{2+}$ only increased 12.8 -fold. This dramatic difference in affinity may be due to $\mathrm{Cd}^{2+}$ having a marked preference for aspartate and glutamate residues, which are not included in the hypothesized binding residues, but are plentiful across CCSBPII's surface. ${ }^{53} \mathrm{Cd}^{2+}$ is relatively flexible with its geometric preference compared to the other metal ions tested here. In fact, $\mathrm{Cd}^{2+}$ has been found in numerous crystal structures of metalloproteins in incompletely formed trigonal planar and tetrahedral complexes, ${ }^{52}$ so gross adventitious binding may be occurring at sites across CCSBPII that may not quench tryptophan fluorescence, similar to one SBP from Listeria monocytogenes (PDB ID: 5JPD). In such circumstances, $x$-ray crystallography is a better choice than microITFQ-LTA for study numerous interactions simultaneously.
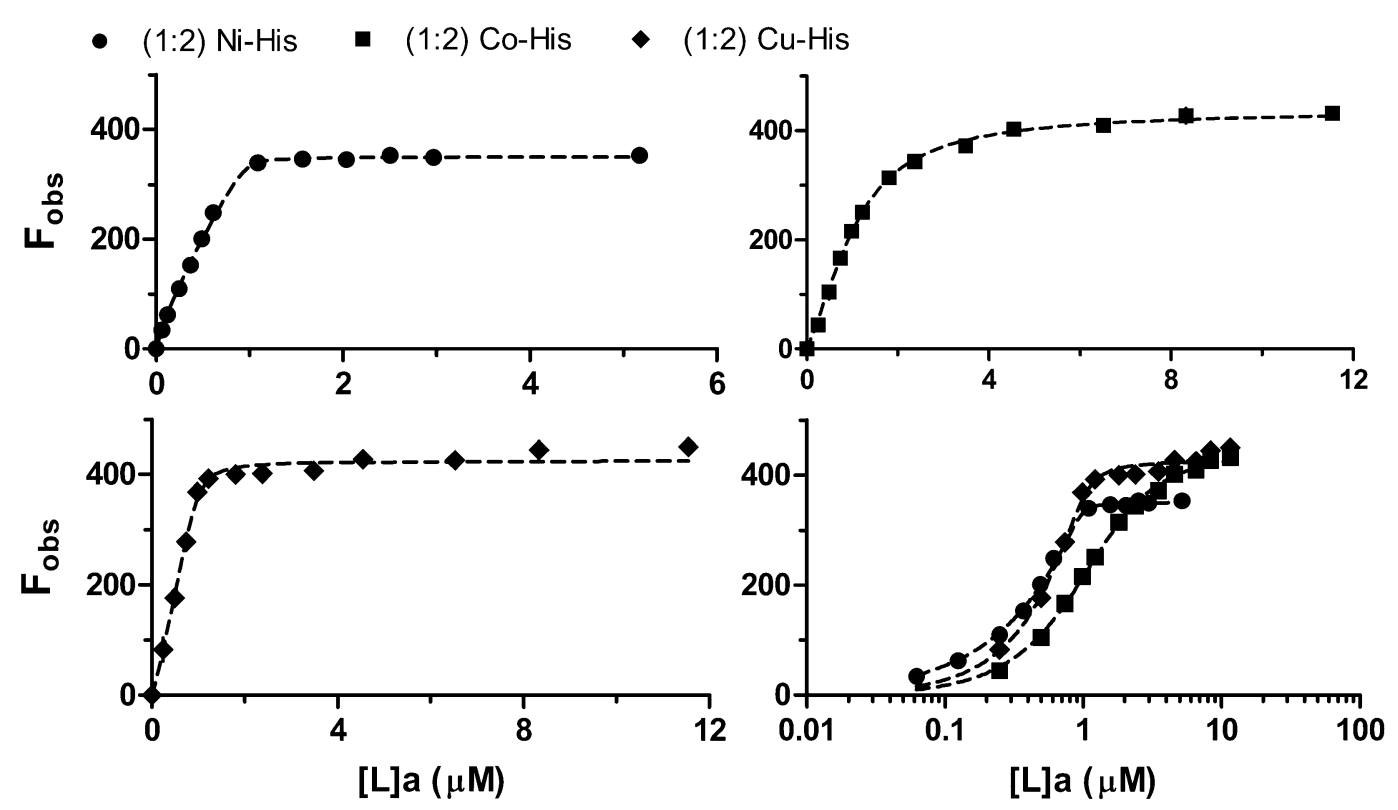

Figure 7. Binding curves for MHC $\rightarrow C C S B P I$ titrations in $10 \mathrm{mM}$ HEPES pH 7.2, where MHC represents a metalhistidine complex prepared with a 1:2 stoichiometric ratio: nickel-histidine or $\mathrm{Ni}$-His (circles), cobalt-histidine or CoHis (squares), and copper-histidine or Cu-His (diamonds). To compare the three, a plot with a logarithmic $\mathrm{x}$-axis is provided for comparison (bottom-right panel).

The apparent ability for CCSBPII to bind $\mathrm{Cu}^{2+} 1.4$-fold more tightly than the hypothesized target $\mathrm{Ni}^{2+}$ and 2.5-fold more tightly than $\mathrm{Co}^{2+}$ is a clear demonstration of an issue bacteria face: mismetallation. ${ }^{54,55}$ The ana operon (Supplementary Figure 1) from which CcSBPII is encoded from also encodes for enzymes predicted to require nickel- and cobalt-based co-factors, supporting the notion that the target is $\mathrm{Ni}^{2+}$ and likely $\mathrm{Co}^{2+}$ too. To improve $\mathrm{Ni}^{2+}$ selectivity, $\mathrm{Ni}$-BPs have been reported to use opine metallophores akin to siderophores for iron species, suggesting that CCSBPII may also naturally use chelators. These opine metallophores are comprised of a histidine connected to a pyruvate or an $\alpha$ ketoglutarate, and Ni-BP crystal structures have been solved with nickel-histidine complexes inside the binding cavities, so we chose to prepare 1:2 stoichiometric ligand solutions of $\mathrm{Cu}^{2+}, \mathrm{Ni}^{2+}$, and $\mathrm{Co}^{2+}$ with $\mathrm{L}-$ 
histidine to titrate into CCSBPII in $10 \mathrm{mM}$ HEPES pH 7.2 (Figure 7). Using Equation (6) with the KK method and inclusion of $\mathrm{n}$, we observed substantial changes in $K_{\mathrm{D}}$, summarized in Table 2 . In all cases $\mathrm{n}$ is near 1 , suggesting a one-binding site model is appropriate. In the absence of histidine, the observed order of affinity from weakest to strongest binding was $\mathrm{Co}^{2+}<\mathrm{Ni}^{2+}<\mathrm{Cu}^{2+}$; in its presence it was $\mathrm{Co}^{2+}<\mathrm{Cu}^{2+}<\mathrm{Ni}^{2+}$. Specifically, by introducing histidine, $K_{\mathrm{D}}$ decreased 37.8 -fold for $\mathrm{Co}^{2+}, 236.7$-fold for $\mathrm{Cu}^{2+}$, and a remarkable 2728.4 -fold for $\mathrm{Ni}^{2+}$ to obtain a $K_{\mathrm{D}}$ of $4.4 \mathrm{nM}$ with a Cl of 1.3 to $8.8 \mathrm{nM}$. CCSBPII binds 11.6 -fold more tightly to a nickel-histidine complex than $C j N i k Z$, reported earlier in this study to have a $K_{\mathrm{D}}$ of $51 \mathrm{nM}$. Further testing reveals that the imidazole ring of histidine contributes the most to the decrease in $K_{\mathrm{D}}$ compared to glycine (the backbone), but the presence of both imidazole and glycine is not sufficient enough for improving the binding affinity and selectivity for $\mathrm{Ni}^{2+}$ (Figure 8). This indicates it is specifically the histidine molecule, not just its components, that is required to potentially counter mismetallation by $\mathrm{Cu}^{2+}$. However, it is unclear whether CCSBPII is binding to a nickel-histidine complex that has a 1:1 stoichiometry like that found in CjNikZ, or 1:2 like those found in YpYntA or SaNikA. ${ }^{28}$

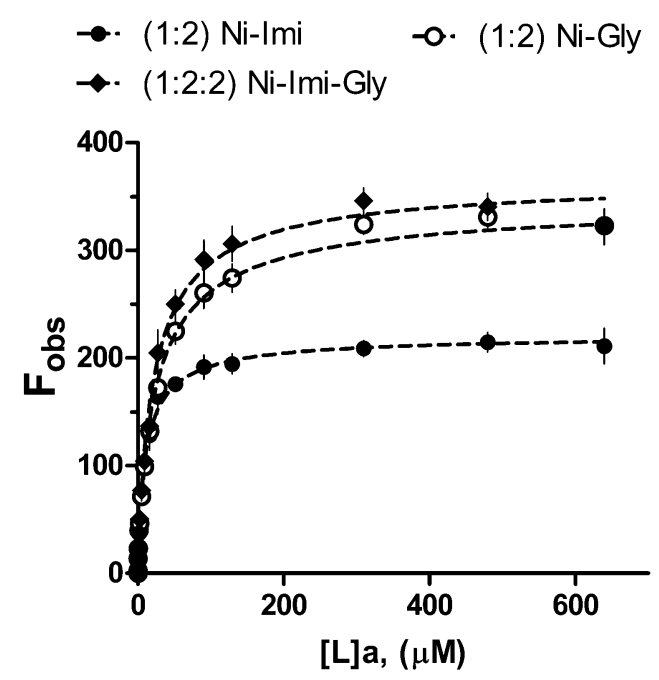

Figure 8. Binding curves for $C \rightarrow C C S B P I I$ titrations in $10 \mathrm{mM}$ HEPES $\mathrm{pH} 7.2$, where $C$ represents a nickel in complex with imidazole (1:2, Ni-Imi, filled circles), glycine (1:2, Ni-Gly, empty circles), and both (1:2:2, Ni-Imi-Gly, diamond).

\section{Limitations of the method}

The microlTFQ-LTA has several limitations that ought to be considered during experimental design and data analysis. First, the user must estimate the magnitude of $[L]_{a}$ that will lead to maximal protein fluorescence quenching and prepare their ligand solution accordingly. If the user does not reach saturation in the titration range tested, best-fit parameter values will be poorly determined and lead to incorrect conclusions about the protein's binding profile. We recommend a titration range over 3 orders of magnitude with a plateau reached in the largest order of magnitude; or alternatively, when the $F_{\text {obs }}$ value ceases to increase with differences within 1 standard deviation for the last three data points. For example, in Figure 4, we did not observe saturation of CCSBPII by $\mathrm{Ni}^{2+}$ in the presence of 100 and 1000 $\mathrm{mM} \mathrm{NaCl}$. The corresponding best-fit parameter values therefore need to be interpreted with caution. Additionally, the beginning of the binding curve has a larger effect on the $K_{D}$ value, so it is important for users to have enough titration steps to accurately fit the model.

Second, $\mathrm{Cu}^{2+}$ is known to undergo a redox reaction with the hydroxyl group of HEPES to form $\mathrm{Cu}^{+}{ }^{56}$ We attempted to measure the IFE this may cause by titrating $\mathrm{Cu}^{2+}$ (same preparation and concentrations used in the $\mathrm{Cu}^{2+} \rightarrow C \mathrm{CSBPII}$ assay) into $10 \mathrm{mM}$ HEPES pH 7.2 without protein and did not observe anything different from titrating the buffer alone (data not shown). $\mathrm{Cu}^{+}$prefers tetrahedral 
geometries and lower coordination numbers, ${ }^{57}$ and our observation that CCSBPII has a low affinity for $\mathrm{Zn}^{2+}$, which has a preferred tetrahedral geometry in protein structures, suggests that it is unlikely CcSBPII would specifically bind to $\mathrm{Cu}^{+}$as the major copper species with a $K_{\mathrm{D}}$ value $(8.54 \mu \mathrm{M})$ less than that of $\mathrm{Ni}^{2+}(12.00$ $\mu \mathrm{M})$. Additionally, $\mathrm{Cu}^{+}$requires stabilization through interactions with alkenes and amines. This means its concentrations can be very low (viz. its presence is negligible) without catalysts or additional chemicals to drive the redox reaction forward and maintain its presence..$^{58}$ In our purification and activity buffers, we did not add such components. Stabilization of $\mathrm{Cu}^{+}$by adventitious binding to the protein's amine groups could occur, especially for micromolar and nanomolar $K_{\mathrm{D}}$ interactions as observed here, ${ }^{59}$ but this phenomenon is poorly studied and does not nullify the usefulness of well documented methods for estimating $K_{D}$ values. Nevertheless, we caution future users of the microlTFQ-LTA to carefully analyze data involving $\mathrm{Cu}^{2+}$, especially when additional buffering components are introduced that may stabilize the $\mathrm{Cu}^{+}$ species. To better characterize the redox state of copper bound to CCSBPII, electron paramagnetic resonance (EPR) spectroscopy and nuclear magnetic resonance (NMR) are recommended. ${ }^{60}$

Third, the models presented here in Equations (6) and (7) are not appropriate for definitively elucidating mismetallation, and therefore directly studying the competition between metal ions. Our study of histidine's effect on CCSBPII's ability to bind to $\mathrm{Ni}^{2+}$ compared to $\mathrm{Cu}^{2+}$ (Figure 7) must be further explored using appropriate models. ${ }^{59,61}$ Similarly, studying the binding stoichiometry for metal complexes (i.e. histidine with $\mathrm{Ni}^{2+}, \mathrm{Co}^{2+} \mathrm{Cu}^{2+}$ ) cannot simply use the Hill Coefficient $\mathrm{n}$ appended to Equations (6) and (7) to definitively elucidate the ratio of protein to metal to complexing agent. We mostly observed $\mathrm{n} \approx 1$ meaning that a one-site binding model could be true in many cases, but further research is needed using other binding models, ${ }^{59}$ or ITC and $\mathrm{x}$-ray crystallography ${ }^{42}$ to support these findings.

Lastly, we note that the position of tryptophan residues in the protein structure should be inspected to determine if they are solvent exposed and protruding into the metal-binding site as this could lead to collisional quenching in addition to the intramolecular self-quenching underpinning the microlTFQLTA described here. Such a scenario would lead to a measured fluorescence quenching that would be a combination of the two phenomena, thus overestimating the association phenomenon. Structural data for proteins can be used to inspect the spatial location of the tryptophan residues computationally or experimentally. ${ }^{62,63}$ Correcting for collisional quenching is described elsewhere. ${ }^{9}$ For CjNikZ and CcSBPII, our structural analysis (data not shown) revealed their tryptophan residues are buried in the hydrophobic core.

\section{Conclusion}

The validity of the microIPFQ-LTA method proposed in this study was confirmed by using it to determine the $K_{\mathrm{D}}$ value for $\mathrm{Ni}^{2+} \rightarrow C j \mathrm{NikZ}$ and (1:2) Ni-His $\rightarrow C j \mathrm{NikZ}$ binding events. We were able to closely replicate existing $K_{D}$ values from literature and support crystal structure data. We then tested this method on CCSBPII, an uncharacterized putative Ni-BP from C. carboxidivorans, to demonstrate how a series of microITFQ-LTAs could be designed to study the effect of buffer composition on $\mathrm{Ni}^{2+} \rightarrow C c S B P I I$ binding and how CCSBPII interacts with various metal ions and complexes.

Approximately 240 replicates were conducted using the microlTFQ-LTA in this study. The time required to process a single 96 -well plate (i.e. one set of titrations manually by multichannel pipette) was $1.5 \mathrm{hrs}$. 240 replicates would have required $>350 \mathrm{hrs}$ (at least two weeks straight) if performed on a onecuvette-per-run spectrofluorometer; ITC would have taken substantially longer. While dedicated spectrofluorometers and ITC can provide more in-depth and more comprehensive analyses of binding events, high-throughput methods like the microlTFQ-LTA are crucial for exploring protein libraries with large buffer and ligand screens. 


\title{
Competing interest statement
}

511

512

513

514

515

516

517

518

519

520

521

522

523

524

525

526

527

528

529

530

531

532

533

534

535

536

537

538

539

540

541

542

543

544

545

546

547

548

549

550

551

552

553

554

555

556

557

558

The authors declare they have no competing financial interests.

\begin{abstract}
Abbreviations
LB - Luria-Bertani, TB - terrific broth, ACN - acetonitrile, TFA - trifluoracetic acid, HEPES - (4-(2hydroxyethyl)-1-piperazineethanesulfonic acid), IPTG - isopropyl $\beta$-D-1-thiogalactopyranosid, TCEP tris(2-carboxyethyl)phosphine, MWCO - molecular weight cut-off, NTA - nitriloacetic acid, SDS-PAGE sodium dodecyl sulfate polyacrylamide gel electrophoresis, TEV - tobacco endomosaic virus, FPLC - fast performance liquid chromatography, IMAC - immobilized metal-affinity chromatography, aEX - anion exchange chromatography, ITFQ-LTA - intrinsic tryptophan fluorescence quenching ligand titration assay, ICP-MS - inductively coiled plasma mass spectrometry, MALDI-ToF - matrix assisted laser desorptionionization time-of-flight, CD - circular dichroism, PDB - Protein Data Bank, IFE - Inner Filter Effect, NLLS non-linear least squares, ITC - isothermal titration calorimetry
\end{abstract}

\section{Acknowledgements}

The authors thank (1) Anna Khusnutdinova, Robert Flick, and Chao Chen for advice on experimental design and data processing, (2) Dr. Bridget A. Bergquist and Joan de Vera for access to and training on their Thermo-Fisher X-Series 2 ICP-MS in the Department of Earth Sciences at the University of Toronto, and (3) Dr. Walid Houry, Vaibhav Bhandari, and Elisa Leung for access to and training on the Bruker MALDIToF Biotyper and the JASCO J-800 spectropolarimeter in the Department of Biochemistry at the University of Toronto. This work was supported by the Ontario Ministry of Economic Development, Job Creation and Trade through the Elements of Bio-mining ORF-RE program. PD is a recipient of an Ontario Graduate Scholarship.

\section{References}

1. Duff, M. R., Grubbs, J. \& Howell, E. E. Isothermal titration calorimetry for measuring macromolecule-ligand affinity. J. Vis. Exp. (2011). doi:10.3791/2796

2. Broecker, J., Vargas, C. \& Keller, S. Revisiting the optimal c value for isothermal titration calorimetry. Anal. Biochem. (2011). doi:10.1016/j.ab.2011.07.027

3. Riihimäki, L. et al. Microplate screening assay for binding of ligands to bovine or reindeer $\beta$-lactoglobulins. J. Biochem. Biophys. Methods (2006). doi:10.1016/j.jbbm.2006.03.019

4. Bodenreider, C. et al. A fluorescence quenching assay to discriminate between specific and nonspecific inhibitors of dengue virus protease. Anal. Biochem. (2009). doi:10.1016/j.ab.2009.08.013

5. Yuan, S. et al. Structure-affinity relationship of the binding of phenolic acids and their derivatives to bovine serum albumin. Food Chem. (2019). doi:10.1016/j.foodchem.2018.11.060

6. Michon, J., Frelon, S., Garnier, C. \& Coppin, F. Determinations of uranium(VI) binding properties with some metalloproteins (transferrin, albumin, metallothionein and ferritin) by fluorescence quenching. J. Fluoresc. (2010). doi:10.1007/s10895-009-0587-3

7. Coppin, F., Michon, J., Garnier, C. \& Frelon, S. Fluorescence quenching determination of uranium (VI) binding properties by two functional proteins: Acetylcholinesterase (AChE) and vitellogenin (Vtg). $J$. Fluoresc. (2015). doi:10.1007/s10895-015-1536-y

8. Ghisaidoobe, A. B. T. \& Chung, S. J. Intrinsic tryptophan fluorescence in the detection and analysis of proteins: A focus on förster resonance energy transfer techniques. International Journal of Molecular Sciences (2014). doi:10.3390/ijms151222518

9. Van De Weert, M. \& Stella, L. Fluorescence quenching and ligand binding: A critical discussion of a popular 
methodology. J. Mol. Struct. (2011). doi:10.1016/j.molstruc.2011.05.023

10. Callis, P. R. B. T.-M. in E. 1La and 1Lb transitions of tryptophan: Applications of theory and experimental observations to fluorescence of proteins. in Flourescence Spectroscopy 278, 113-150 (Academic Press, 1997).

11. Chen, Y. \& Barkley, M. D. Toward understanding tryptophan fluorescence in proteins. Biochemistry (1998). doi:10.1021/bi980274n

12. Royer, C. A. Probing protein folding and conformational transitions with fluorescence. Chemical Reviews (2006). doi:10.1021/cr0404390

13. Callis, P. R. Binding phenomena and fluorescence quenching. I: Descriptive quantum principles of fluorescence quenching using a supermolecule approach. J. Mol. Struct. (2014). doi:10.1016/j.molstruc.2014.04.050

14. Callis, P. R. Binding phenomena and fluorescence quenching. II: Photophysics of aromatic residues and dependence of fluorescence spectra on protein conformation. J. Mol. Struct. (2014).

doi:10.1016/j.molstruc.2014.04.051

15. Hulme, E. C. \& Trevethick, M. A. Ligand binding assays at equilibrium: Validation and interpretation. British Journal of Pharmacology (2010). doi:10.1111/j.1476-5381.2009.00604.x

16. Pollard, T. D. A Guide to Simple and Informative Binding Assays. Mol. Biol. Cell (2010). doi:10.1091/mbc.e10-08-0683

17. Locher, K. P. Mechanistic diversity in ATP-binding cassette (ABC) transporters. Nat. Struct. Mol. Biol. 23, 487-493 (2016).

18. Navarro, C., Wu, L. -F \& Mandrand-Berthelot, M. -A. The nik operon of Escherichia coli encodes a periplasmic binding-protein-dependent transport system for nickel. Mol. Microbiol. (1993). doi:10.1111/j.1365-2958.1993.tb01247.x

19. De Pina, K. et al. Purification and Characterization of the Periplasmic Nickel-Binding Protein NikA of Escherichia coli K12. Eur. J. Biochem. (1995). doi:10.1111/j.1432-1033.1995.0857p.x

20. Sebbane, F., Mandrand-Berthelot, M. A. \& Simonet, M. Genes encoding specific nickel transport systems flank the chromosomal urease locus of pathogenic yersiniae. J. Bacteriol. (2002). doi:10.1128/JB.184.20.5706-5713.2002

21. Heddle, J., Scott, D. J., Unzai, S., Park, S. Y. \& Tame, J. R. H. Crystal Structures of the Liganded and Unliganded Nickel-binding Protein NikA from Escherichia coli. J. Biol. Chem. (2003). doi:10.1074/jbc.M307941200

22. Cherrier, M. V. et al. Crystallographic and spectroscopic evidence for high affinity binding of FeEDTA(H2O)to the periplasmic nickel transporter NikA. J. Am. Chem. Soc. (2005). doi:10.1021/ja0518530

23. Rowe, J. L., Starnes, G. L. \& Chivers, P. T. Complex transcriptional control links NikABCDE-dependent nickel transport with hydrogenase expression in Escherichia coli. J. Bacteriol. (2005). doi:10.1128/JB.187.18.63176323.2005

24. Cherrier, M. V., Cavazza, C., Bochot, C., Lemaire, D. \& Fontecilla-Camps, J. C. Structural characterization of a putative endogenous metal chelator in the periplasmic nickel transporter NikA. Biochemistry (2008). doi:10.1021/bi801051y

25. Cherrier, M. V. et al. The structure of the periplasmic nickel-binding protein NikA provides insights for artificial metalloenzyme design. J. Biol. Inorg. Chem. (2012). doi:10.1007/s00775-012-0899-7

26. Lebrette, H., lannello, M., Fontecilla-Camps, J. C. \& Cavazza, C. The binding mode of Ni-(L-His)2 in NikA revealed by X-ray crystallography. J. Inorg. Biochem. (2013). doi:10.1016/j.jinorgbio.2012.12.010

27. Howlett, R. M., Hughes, B. M., Hitchcock, A. \& Kelly, D. J. Hydrogenase activity in the foodborne pathogen Campylobacter jejuni depends upon a novel ABC-type nickel transporter (NikZYXWV) and is SlyDindependent. Microbiol. (United Kingdom) (2012). doi:10.1099/mic.0.054130-0

28. Lebrette, H. et al. Promiscuous nickel import in human pathogens: Structure, thermodynamics, and evolution of extracytoplasmic nickel-binding proteins. Structure (2014). doi:10.1016/j.str.2014.07.012

29. Jubier-Maurin, V. et al. Identification of the nik gene cluster of Brucella suis: Regulation and contribution to urease activity. J. Bacteriol. (2001). doi:10.1128/JB.183.2.426-434.2001

30. Lebrette, H. et al. Novel insights into nickel import in Staphylococcus aureus: The positive role of free histidine and structural characterization of a new thiazolidine-type nickel chelator. Metallomics (2015). doi:10.1039/c4mt00295d 
Benoit, S. L., Seshadri, S., Lamichhane-Khadka, R. \& Maier, R. J. Helicobacter hepaticus NikR controls urease and hydrogenase activities via the NikABDE and HH0418 putative nickel import proteins. Microbiol. (United Kingdom) (2013). doi:10.1099/mic.0.062976-0

32. Shaik, M. M., Cendron, L., Salamina, M., Ruzzene, M. \& Zanotti, G. Helicobacter pylori periplasmic receptor CeuE (HP1561) modulates its nickel affinity via organic metallophores. Mol. Microbiol. (2014). doi:10.1111/mmi.12487

33. Park, K. S. et al. Genetic characterization of DNA region containing the trh and ure genes of Vibrio parahaemolyticus. Infect. Immun. (2000). doi:10.1128/IAI.68.10.5742-5748.2000

34. Hughes, A., Wilson, S., Dodson, E. J., Turkenburg, J. P. \& Wilkinson, A. J. Crystal structure of the putative peptide-binding protein AppA from Clostridium difficile. Acta Crystallogr. Sect. F 75, 246-253 (2019).

35. Almagro Armenteros, J. J. et al. SignalP 5.0 improves signal peptide predictions using deep neural networks. Nat. Biotechnol. (2019). doi:10.1038/s41587-019-0036-z

36. Kemmer, G. \& Keller, S. Nonlinear least-squares data fitting in Excel spreadsheets. Nat. Protoc. (2010). doi:10.1038/nprot.2009.182

37. Ferreira, C. M. H., Pinto, I. S. S., Soares, E. V. \& Soares, H. M. V. M. (Un)suitability of the use of pH buffers in biological, biochemical and environmental studies and their interaction with metal ions-a review. RSC Advances (2015). doi:10.1039/c4ra15453c

38. Quinn, C. F., Carpenter, M. C., Croteau, M. L. \& Wilcox, D. E. Isothermal Titration Calorimetry Measurements of Metal lons Binding to Proteins. in Methods in Enzymology (2016). doi:10.1016/bs.mie.2015.08.021

39. Queiroz, H. M. et al. The Samarco mine tailing disaster: A possible time-bomb for heavy metals contamination? Sci. Total Environ. (2018). doi:10.1016/j.scitotenv.2018.04.370

40. Zbacnik, T. J. et al. Role of Buffers in Protein Formulations. Journal of Pharmaceutical Sciences (2017). doi:10.1016/j.xphs.2016.11.014

41. Ugwu, S. O. \& Apte, S. P. The Effect of Buffers on Protein Conformational Stability. Pharmaceutical Technology (2004).

42. Handing, K. B. et al. Characterizing metal-binding sites in proteins with X-ray crystallography. Nat. Protoc. (2018). doi:10.1038/nprot.2018.018

43. Escudero, G., Espinoza, E. \& Rao, F. Chemical Precipitation of Nickel Species from Waste Water. Int. Res. J. Pure Appl. Chem. (2017). doi:10.9734/irjpac/2017/37905

44. Vetting, M. W. et al. Experimental strategies for functional annotation and metabolism discovery: Targeted screening of solute binding proteins and unbiased panning of metabolomes. Biochemistry (2015). doi:10.1021/bi501388y

45. Giuliani, S. E., Frank, A. M. \& Collart, F. R. Functional assignment of solute-binding proteins of $A B C$ transporters using a fluorescence-based thermal shift assay. Biochemistry (2008). doi:10.1021/bi801648r

46. Zerbs, S., Korajczyk, P. J., Noirot, P. H. \& Collart, F. R. Transport capabilities of environmental Pseudomonads for sulfur compounds. Protein Sci. (2017). doi:10.1002/pro.3124

47. Neupane, D. P., Avalos, D., Fullam, S., Roychowdhury, H. \& Yukl, E. T. Mechanisms of zinc binding to the solute-binding protein AztC and transfer from the metallochaperone AztD. J. Biol. Chem. (2017). doi:10.1074/jbc.M117.804799

48. Dong, X. B. et al. Purification and Characterization of a Cadmium-Binding Protein from Lentinula edodes. J. Agric. Food Chem. (2019). doi:10.1021/acs.jafc.8b05924

49. Irving, H. \& Williams, R. J. P. 637. The stability of transition-metal complexes. J. Chem. Soc. (2004). doi:10.1039/jr9530003192

50. Lawrance, G. A. Introduction to Coordination Chemistry. Introduction to Coordination Chemistry (2009). doi:10.1002/9780470687123

51. Dudev, T. \& Lim, C. Tetrahedral vs octahedral zinc complexes with ligands of biological interest: A DFT/CDM study. J. Am. Chem. Soc. (2000). doi:10.1021/ja0010296

52. Barber-Zucker, S., Shaanan, B. \& Zarivach, R. Transition metal binding selectivity in proteins and its correlation with the phylogenomic classification of the cation diffusion facilitator protein family. Sci. Rep. (2017). doi:10.1038/s41598-017-16777-5

53. Jesu Jaya Sudan, R. \& Sudandiradoss, C. Pattern prediction and coordination geometry analysis from cadmium-binding proteins: A computational approach. Acta Crystallogr. Sect. D Biol. Crystallogr. (2012). 
665

666

667

668

669

670

671

672

673

674

675

676

677

678

679

680

681

682

683

684

685

686

687

688

689

690

691

692

693

694

695

696

697

698

699
doi:10.1107/S0907444912028776

54. Waldron, K. J. \& Robinson, N. J. How do bacterial cells ensure that metalloproteins get the correct metal? Nature Reviews Microbiology (2009). doi:10.1038/nrmicro2057

55. Dudev, T. \& Lim, C. Competition among metal ions for protein binding sites: Determinants of metal ion selectivity in proteins. Chemical Reviews (2014). doi:10.1021/cr4004665

56. Hegetschweiler, K. \& Saltman, P. Interaction of Copper(II) with N-(2-Hydroxyethyl)piperazine-N'ethanesulfonic Acid (HEPES). Inorg. Chem. (1986). doi:10.1021/ic00221a028

57. Conry, R. R. Copper: Inorganic \& Coordination ChemistryBased in part on the article Copper: Inorganic \& Coordination Chemistry by Rebecca R. Conry \& Kenneth D. Karlin which appeared in the Encyclopedia of Inorganic Chemistry, First Edition . . in Encyclopedia of Inorganic and Bioinorganic Chemistry (2011). doi:10.1002/9781119951438.eibc0050

58. Navon, N. et al. Design of ligands that stabilize $\mathrm{Cu}(\mathrm{I})$ and shift the reduction potential of the Cull/I couple cathodically in aqueous solutions. Inorg. Chem. (1999). doi:10.1021/ic9812048

59. Xiao, Z. \& Wedd, A. G. The challenges of determining metal-protein affinities. Natural Product Reports (2010). doi:10.1039/b906690j

60. Crichton, R. R. Methods to Study Metals in Biological Systems. in Biological Inorganic Chemistry (2008). doi:10.1016/b978-044452740-0.50006-5

61. Bougie, I., Charpentier, S. \& Bisaillon, M. Characterization of the metal ion binding properties of the hepatitis C virus RNA polymerase. J. Biol. Chem. (2003). doi:10.1074/jbc.M209785200

62. Strohalm, M., Šantrůček, J., Hynek, R. \& Kodíček, M. Analysis of tryptophan surface accessibility in proteins by MALDI-TOF mass spectrometry. Biochem. Biophys. Res. Commun. (2004).

doi:10.1016/j.bbrc.2004.08.217

63. Waterhouse, A. et al. SWISS-MODEL: Homology modelling of protein structures and complexes. Nucleic Acids Res. (2018). doi:10.1093/nar/gky427

64. Solovyev, V. \& Salamov, A. Automatic Annotation of Microbial Genomes and Metagenomic Sequences. in Metagenomics and its Applications in Agriculture, Biomedicine and Environmental Studies (2011).

65. Salis, H. M. The ribosome binding site calculator. in Methods in Enzymology (2011). doi:10.1016/B978-012-385120-8.00002-4

66. Mukherjee, S. \& Sengupta, S. Riboswitch Scanner: An efficient pHMM-based web-server to detect riboswitches in genomic sequences. Bioinformatics (2016). doi:10.1093/bioinformatics/btv640

67. Edgar, R. C. MUSCLE: Multiple sequence alignment with high accuracy and high throughput. Nucleic Acids Res. (2004). doi:10.1093/nar/gkh340

68. Wiedemann, C., Bellstedt, P. \& Görlach, M. CAPITO - A web server-based analysis and plotting tool for circular dichroism data. Bioinformatics (2013). doi:10.1093/bioinformatics/btt278 


\title{
Supplementary Materials
}

\author{
Appendix A - Additional Protocols
}

\section{Bioinformatics of the ana operon and CCSBPII}

The ana operon of $C$. carboxidivorans was discovered through a blastp search using the CjNikZ open-reading frame (UniProt ID: AOAOM4UJ91), and can be found here: tiny.cc/ccanaoperon. The locations of the promoter, ribosome binding sites, and transcriptional terminator were predicted using BPROM $^{64}$, RBS Calculator ${ }^{65}$, and FindTerm ${ }^{64}$, respectively. The cobalamin riboswitch was predicted using Riboswitch Scanner. ${ }^{66}$ Multiple sequence alignments were made and visualized on JalView using the MUSCLE algorithm with default settings.$^{67}$ The 3D structure of CCSBPII was made using SWISS-MODEL with unliganded CjNikZ as the template (PDB ID: 4OET), and visualized on PyMOL. ${ }^{28,63}$

\section{Matrix-assisted laser desorption/ionization time-of-flight (MALDI-ToF)}

Matrix solution ( $30 \% \mathrm{v} / \mathrm{v}$ ACN, $0.05 \% \mathrm{v} / \mathrm{v}$ TFA , sinapinic acid to saturation) was briefly centrifuged to remove undissolved sinapanic acid, then combined with $1 \mu \mathrm{L}$ of $1 \mathrm{mg} / \mathrm{mL}$ CCSBPII in a 1:1 ratio and applied to a polished steel target plate (Bruker, Part No. 8281817) to dry at room temperature. The Bruker Microflex LT MALDI-ToF was used in the $20-100 \mathrm{kDa}$ range to analyze the ACN:TFA-CCSBPII precipitate by collecting 200 shots with the laser at $85 \%$ power and $60 \mathrm{~Hz}$. Data was analyzed on flexAnalysis v3.4.

\section{Inductively-coiled plasma mass spectrometry (ICP-MS)}

A multi-element standard curve (Millipore Sigma, \#1094800100) was prepared $(0,0.01,0.1,0.5$, $1,5,10,15$, and $20 \mathrm{ppb}$ ) volumetrically in MilliQ water, each containing a final concentration of $2 \%$ TraceMetal $^{\mathrm{TM}}$ Grade $\mathrm{HNO}_{3}$ (ThermoFisher, \#A509P500) and 8 ppb TraceCERT ${ }^{\circledR}$ germanium (Millipore Sigma, \#05419) for the internal standard. CCSBPII samples for metal analysis were prepared in the same manner with a final CCSBPII concentration of $0.67 \mu \mathrm{M}$ (total volume $=3 \mathrm{~mL}$ per CcSBPII sample). For metal analysis, the ICP-MS (Thermo-Fisher X-Series 2) was equilibrated at $20^{\circ} \mathrm{C}$ for $30 \mathrm{~min}$. The system was then flushed with $2 \%$ TraceMetal ${ }^{\mathrm{TM}}$ Grade $\mathrm{HNO}_{3}$ for $30 \mathrm{~min}$ and tuned using the tune A solution (Fisher Scientific, \#NC9063415). Triplicates were acquired and processed automatically by PlasmaLab, then visualized using GraphPad Prism 5.0.

\section{Circular dichroism (CD)}

The JASCO-800 spectropolarimeter was flushed with nitrogen gas (20 L/min) for $15 \mathrm{~min}$ and allowed to equilibrate at $25{ }^{\circ} \mathrm{C}$ with running distilled water before turning the lamp on. A 1-mm quartz cuvette (Millipore Sigma, \#Z800015) was washed with MilliQ water, $70 \% \mathrm{EtOH}, 70 \% \mathrm{HNO}_{3}$, and a final wash with MilliQ water. $1 \mu \mathrm{M}$ of purified CCSBPII in MilliQ water was added to the cuvette and analyzed in triplicate using the Measurement Mode between $180-260 \mathrm{~nm}$ at a $1 \mathrm{~nm}$ interval. The curve was then smoothed using a Savitzky-Golay filter on CAPITO ${ }^{68}$, then visualized using GraphPad Prism 5.0. 


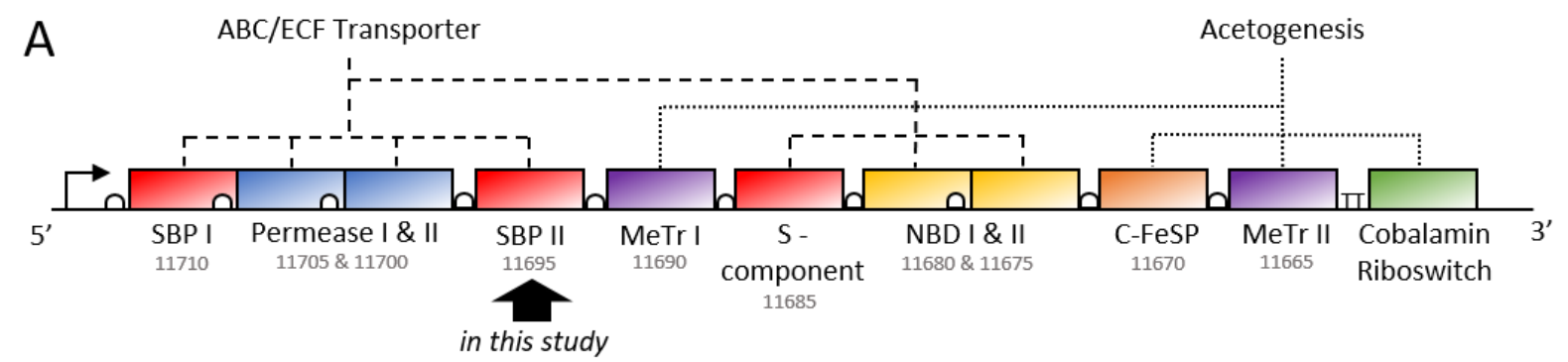

B

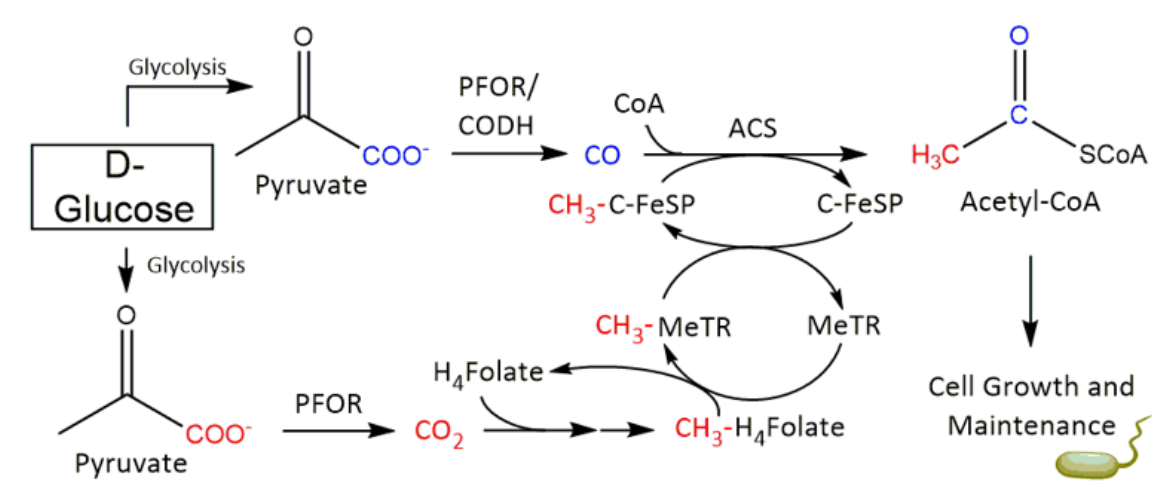

Supplementary Figure 1. Schematic diagram of the ana operon from Clostridium carboxidivorans encoding a potential acetogenesis pathway. (A) The ana operon is comprised of two sets of coding sequences. The first encodes an $A B C$ transporter, which includes two solute binding proteins (SBP I and SBP II), two permease units (Permease I and Permease II), two nucleotidebinding domains (NBD I and NBD II), and an energy-coupling factor S-component. The second encodes acetogenesis-related enzymes, which includes two methyltransferases (MeTR I and MeTR II), one C-FeSP, and a cobalamin riboswitch after the transcriptional terminator (TT). A promoter (right angle arrow) and ribosome binding sites (white semi-circles) indicate where the operon and coding sequences start, respectively. Numbers below coding sequence names are identifiers that can be prefixed with "Ccar_" to locate them in the C. carboxidivorans genome (NZ_CP011803.1). SBP II (CCSBPII) is the protein of interest in this study. (B) Pyruvate is largely derived from glycolysis and possesses a carboxylate group (COO-) noted in blue and red, representing the Western and Eastern branches of the Wood-Ljungdahl pathway, respectively. Numerous enzymes require metal co-factors. PFOR requires a [4Fe-4S] cluster. CODH requires a [3Fe-4S] cluster bridged to a binuclear NiFe cluster. ACS requires a [4Fe-4S] cluster bridged to a binuclear NiNi cluster. C-FeSP requires cobalamin. 


\section{A}
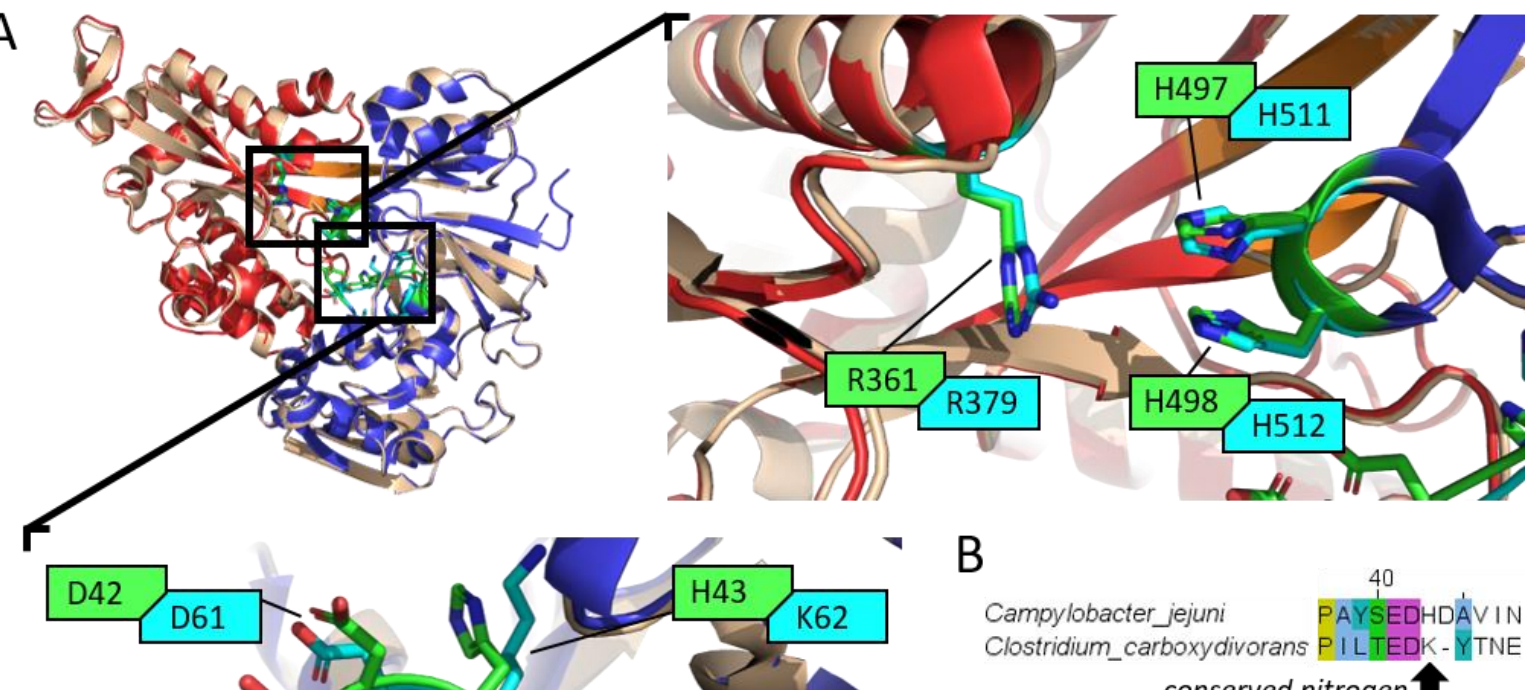

$\mathrm{H} 43$
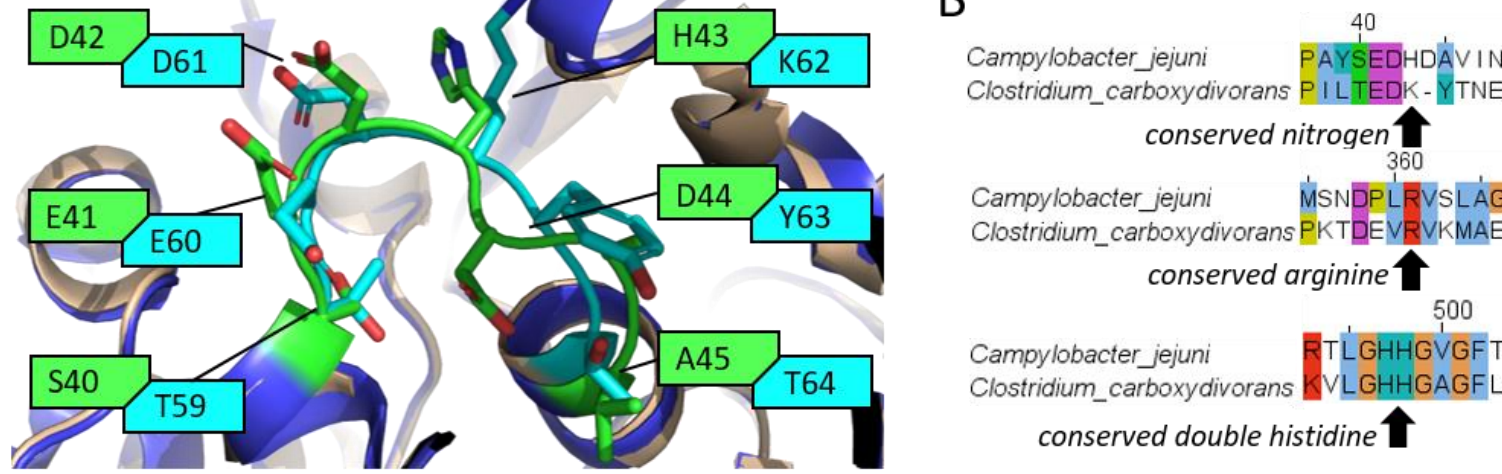

Campylobacter jejuni ṀंSNDPL'RVSLÁG Clostridium_carboxydivorans PKTDEVRVKMAE conserved arginine 1 Clostridium_carboxydivorans.KVLGHHGAGFL conserved double histidine

Supplementary Figure 2. Similarities between CjNikZ and CCSBPII revealed by structural and sequence analysis. (A) A superimposition was made on PyMOL using the unliganded CjNikZ structure (tan, PDB ID: 4OET) and the predicted CcSBPII structure (red - Western lobe, blue - Eastern lobe, orange - hinge region) from SWISS-MODEL with 4OET as the template. The topright panel (RHH cavity) and bottom-left panel (variable loop) are zoom-ins of the superimposition and highlight residue similarities and differences between CjNikZ (green residues) and CCSBPII (cyan residues). (B) A multiple sequence alignment reveals CCSBPII uses $\mathrm{K} 62$ instead of $\mathrm{H} 43$ from $C j N i k Z$, but has conserved binding residues otherwise (black arrows). 


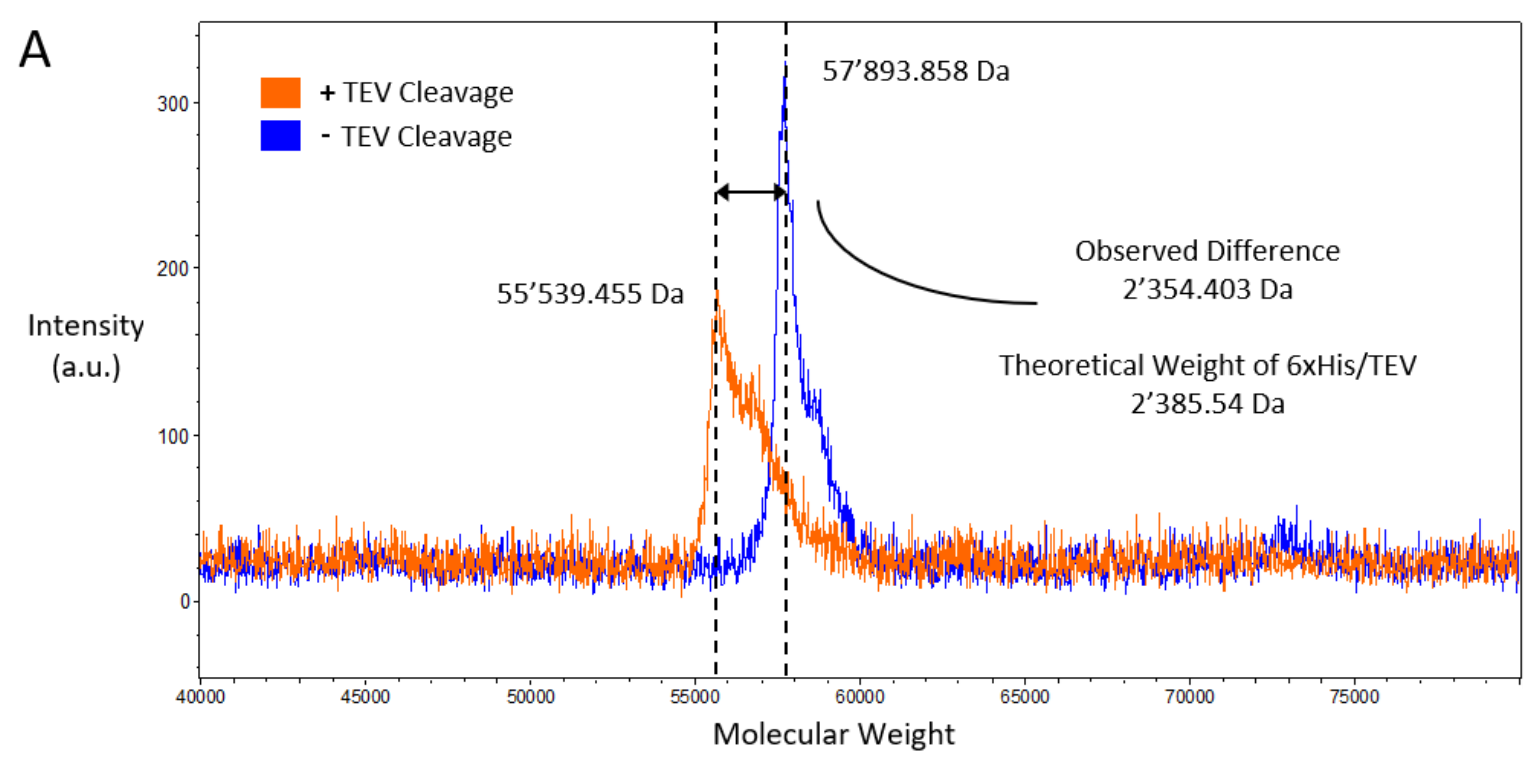

(Da)
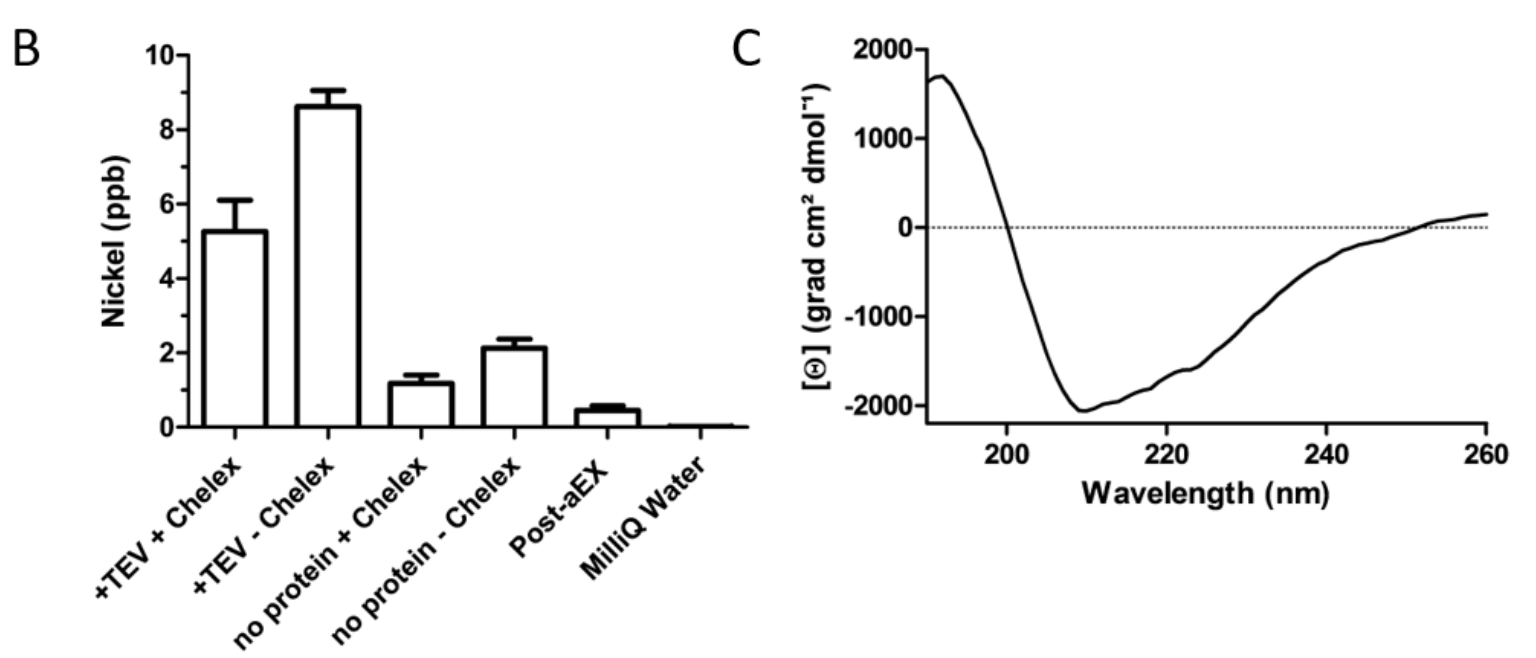

Supplementary Figure 3. Post-purification validation of tag removal, nickel removal, and proper protein folding of purified CCSBPII. (A) MALDI-ToF analysis of protein molecular weight with two samples (orange - addition of TEV, blue - no addition of TEV). Dashed black lines depict the maximum peaks for each sample. (B) ICP-MS analysis of nickel concentration to evaluate the usage of Chelex 100 to remove heavy metals from the Co-IMAC protein eluent, which is collected after applying the cell lysate to the Co-NTA and washing the resin with washing buffer. This protein eluent is combined with TEV for cleavage and dialyzed for 72 hrs with and without Chelex 100, labelled "+TEV +Chelex" and "+TEV -Chelex", respectively. A negative control is included where the cell lysate volume is replaced with binding buffer to determine background nickel contamination by the buffer. No TEV is added to the eluent in this case and is dialyzed in the same fashion, labelled "no protein + Chelex" and "no protein Chelex". CCSBPII after the final $24 \mathrm{hr}$ dialysis, prior to freezing, was also evaluated by ICP-MS and labelled "Post-aEX". (C) CD spectrum of CCSBPII after being blanked with 10 mM HEPES, pH 7.2. 
Supplementary Table 2. CCSBPII purification and purity profile

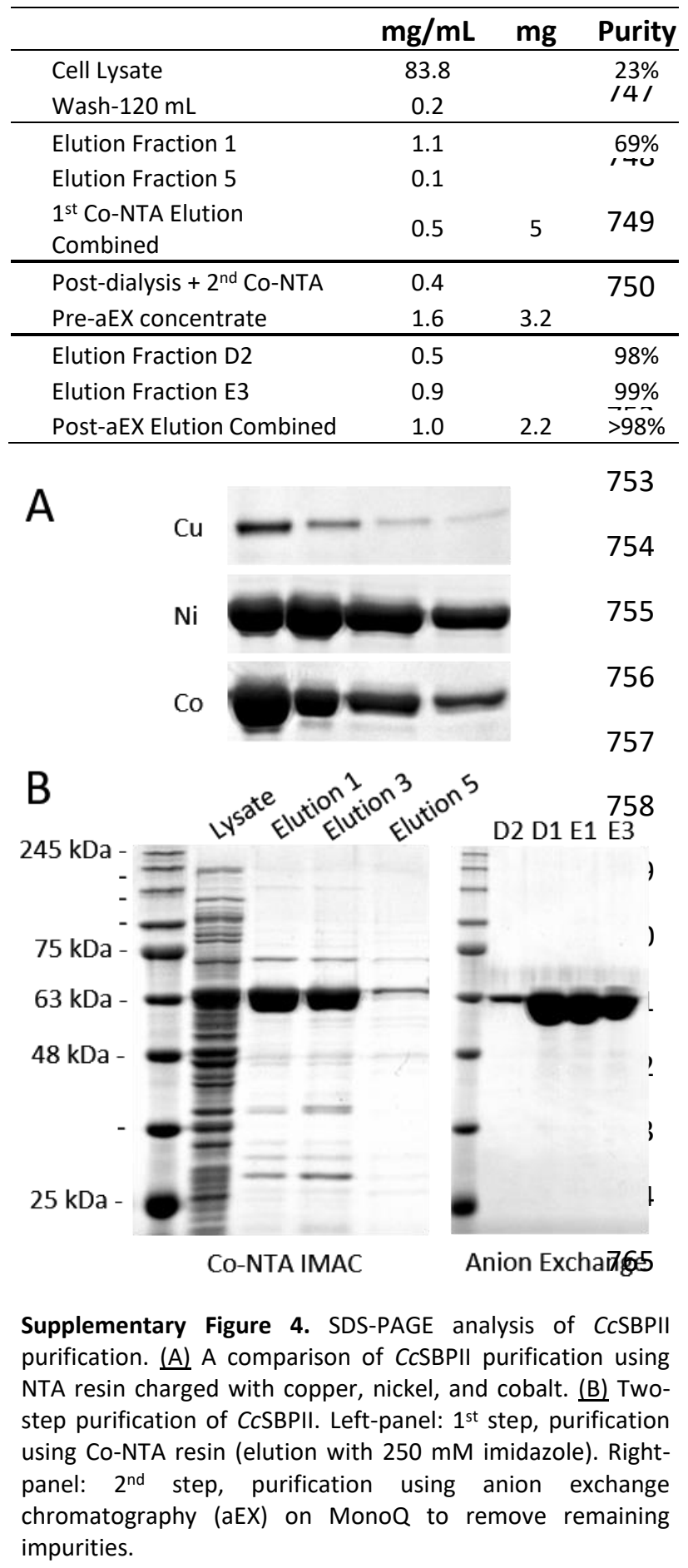



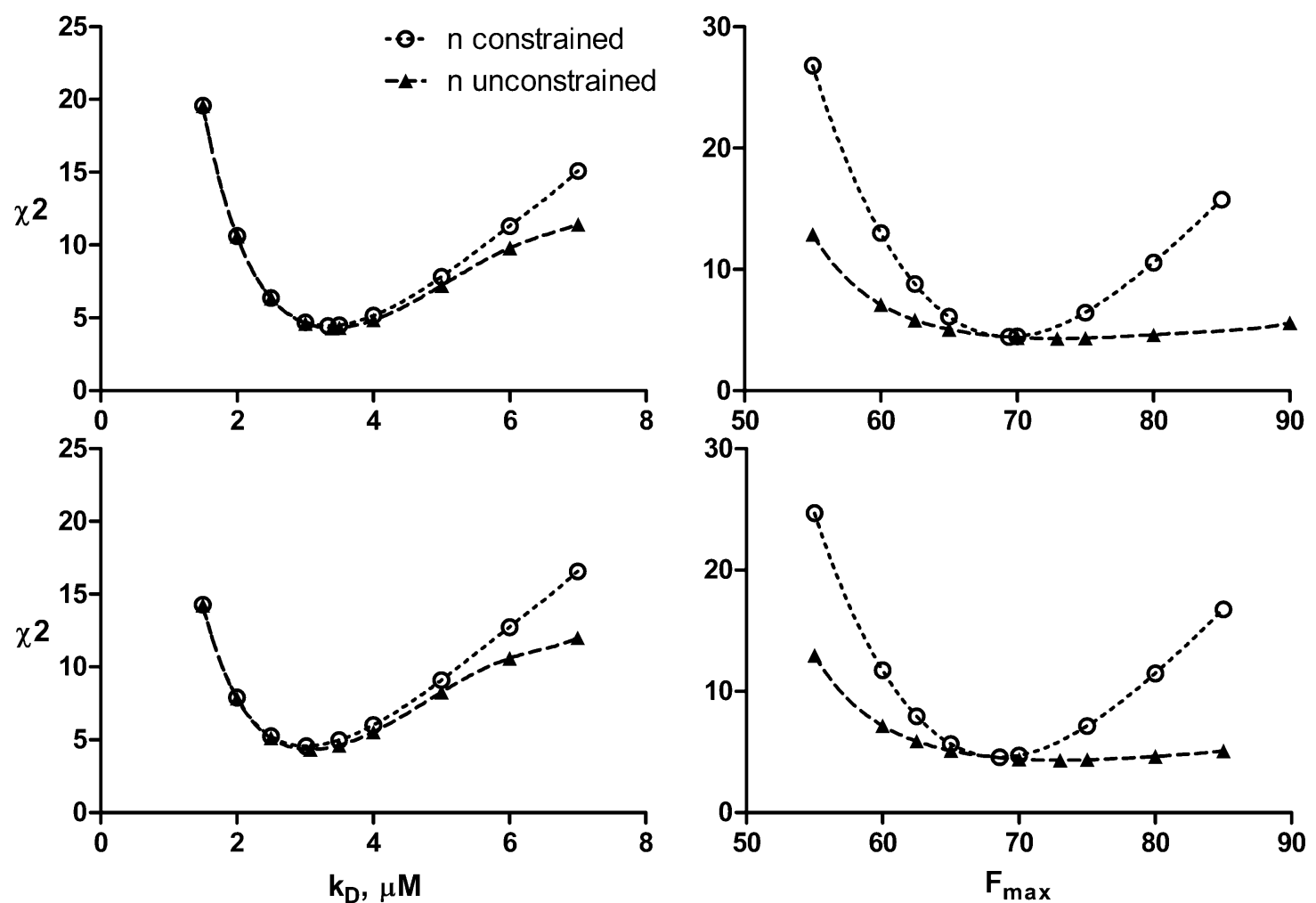

$\mathbf{k}_{\mathrm{D}}, \mu \mathrm{M}$

Supplementary Figure 5. $\chi^{2}$ plots of $\mathrm{Ni}^{2+} \rightarrow C j N i k Z$ to compare how $\mathrm{n}$ constrained $(\mathrm{n}=1$, circles) and $\mathrm{n}$ unconstrained (triangles) affects the behavior of global minima for best-fit $K_{\mathrm{D}}$ and $F_{\max }$ parameters. Top-Left Panel: $\chi^{2}$ vs. $K_{\mathrm{D}}$ using Equation (7). Top-Right Panel: $\chi^{2}$ vs. $F_{\max }$ using Equation (7). Bottom-Left Panel: $\chi^{2}$ vs. $K_{\mathrm{D}}$ using Equation (6). Bottom-Right Panel: $\chi^{2}$ vs. $F_{\max }$ using Equation (6). Curves of best-fit for these plots are polynomial functions generated from Excel, and solved using Wolfram Alpha to generate Cls. These plots are created following instructions described by the KK method. Graph generated on Prism. 

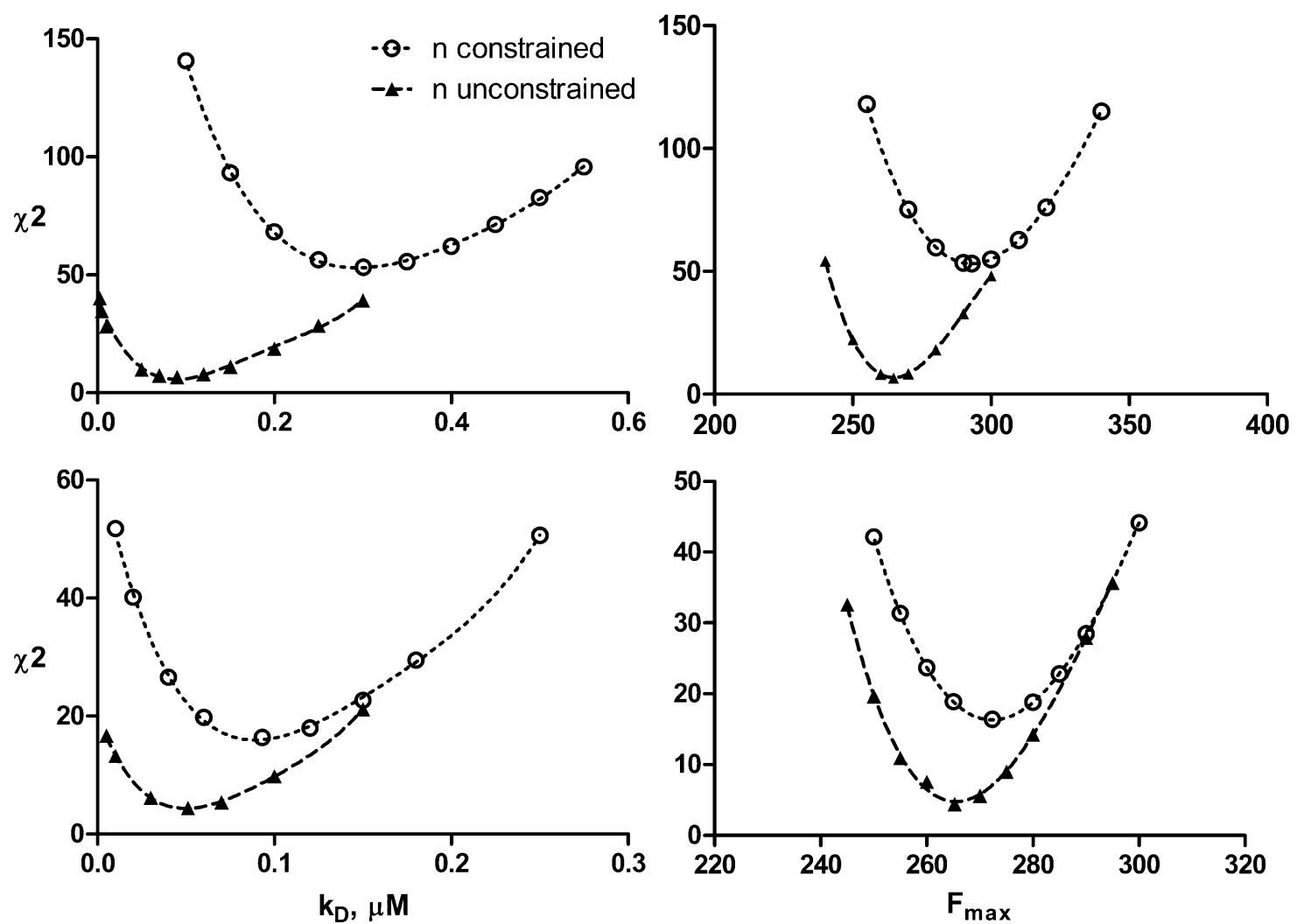

Supplementary Figure 6. $\chi^{2}$ plots of $(1: 2) \mathrm{Ni}$-His $\rightarrow C j N i k Z$ to compare how $n$ constrained $(n=1$, circles) and $n$ unconstrained (triangles) affects the behavior of global minima for best-fit $K_{\mathrm{D}}$ and $F_{\max }$ parameters. Top-Left Panel: $\chi^{2}$ vs. $K_{\mathrm{D}}$ using Equation (7). Top-Right Panel: $\chi^{2}$ vs. $F_{\max }$ using Equation (7). Bottom-Left Panel: $\chi^{2}$ vs. $K_{\mathrm{D}}$ using Equation (6). Bottom-Right Panel: $\chi^{2}$ vs. $F_{\max }$ using Equation (6). Curves of best-fit for these plots are polynomial functions generated from Excel, and solved using Wolfram Alpha to generate $\mathrm{Cls}$. These plots are created following instructions described by the KK method. Graph generated on Prism. 


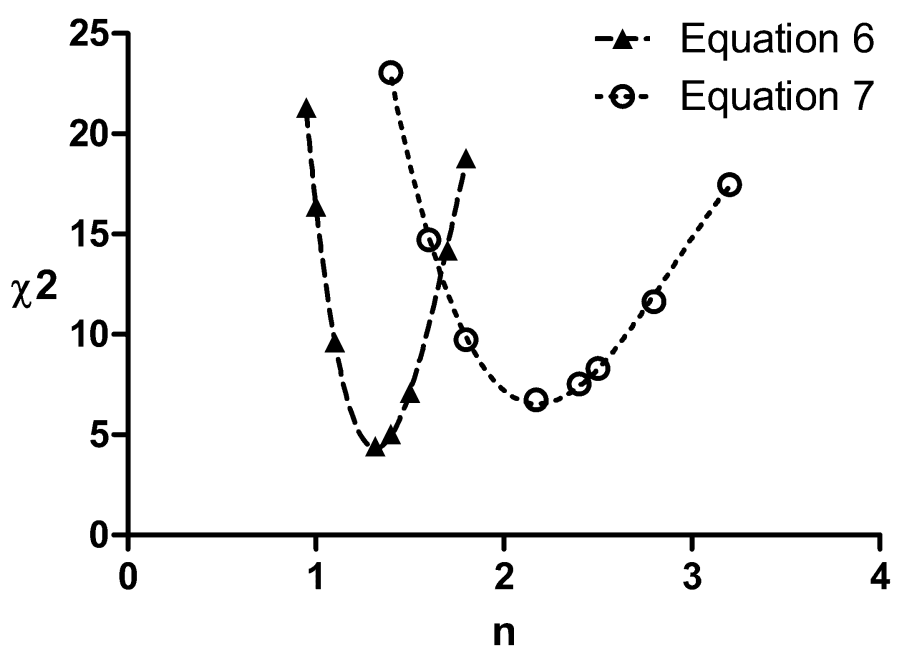

Supplementary Figure 7. $\chi^{2}$ plot of (1:2) Ni-His $\rightarrow C j N i k Z$ to compare how the inclusion of $n$ in Equations (6) and (7) alters the global minimum for the parameter $n$. Curves of best-fit for these plots are polynomial functions generated from Excel, and solved using Wolfram Alpha to generate Cls. These plots are created following instructions described by the KK method. Graph generated on Prism. 


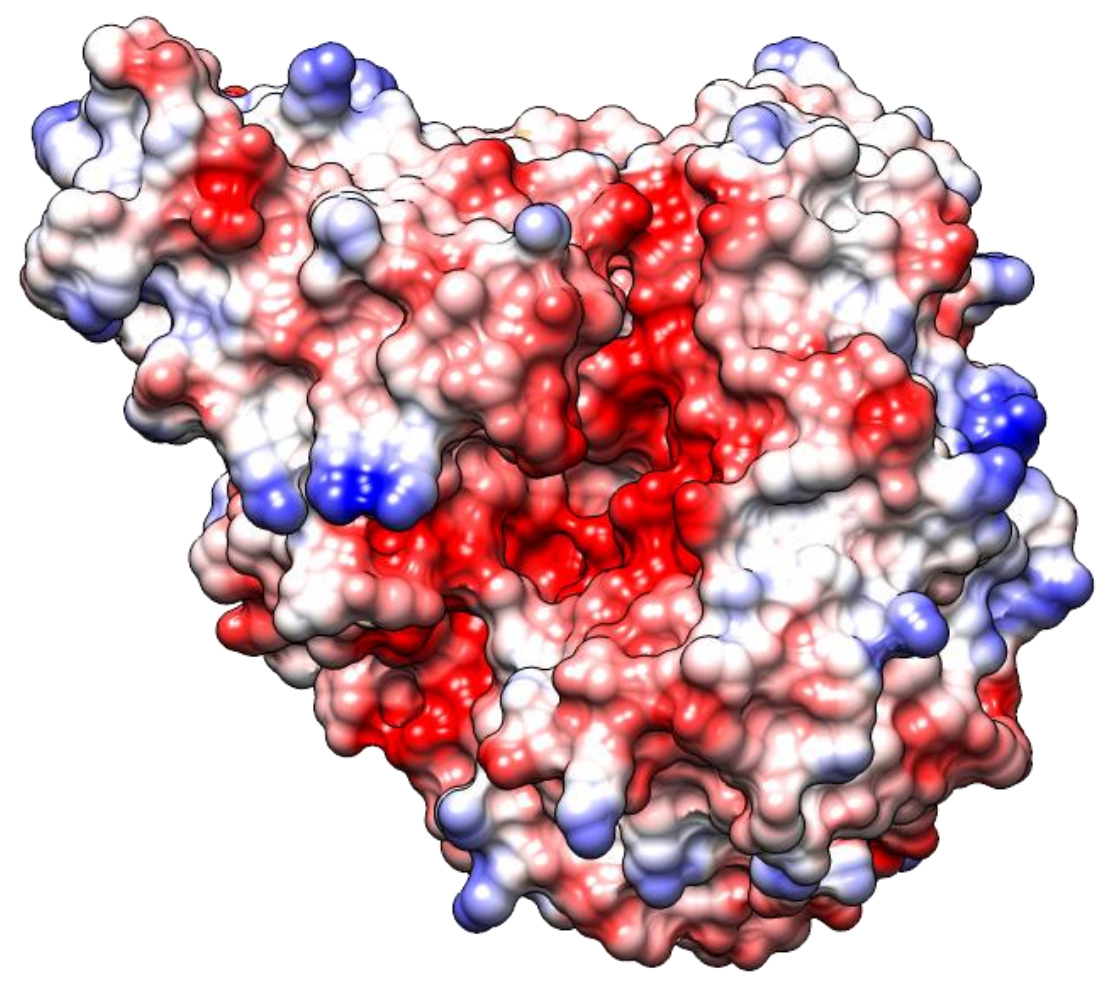

Supplementary Figure 8. Coulombic surface view of CcSBPII (homology model from CjNikZ, PDB ID: 4OET) highlighting regions of positive charge (blue), negative charge (red), and neutral (white). Visualized on Chimera 1.13.1. 